\title{
ON EMBEDDINGS OF FUNCTION CLASSES DEFINED BY CONSTRUCTIVE CHARACTERISTICS
}

\author{
BORIS V. SIMONOV \\ Volgograd State Technical University \\ Volgograd, 400131, Russia \\ E-mail: htf@vstu.ru \\ SERGEY YU. TIKHONOV \\ Centre de Recerca Matematica (CRM) \\ Bellaterra (Barcelona), E-08193, Spain \\ E-mail: tikhonov@mccme.ru
}

\begin{abstract}
In this paper we study embedding theorems for function classes which are subclasses of $L_{p}, 1 \leq p \leq \infty$. To define these classes, we use the notion of best trigonometric approximation as well as that of a $(\lambda, \beta)$-derivative, which is the generalization of a fractional derivative. Estimates of best approximations of transformed Fourier series are obtained.
\end{abstract}

1. Introduction. It is well-known that if $f \in L_{p}, 1 \leq p \leq \infty$, and $\sum_{k=1}^{\infty} k^{r-1} E_{k}(f)_{p}$ $<\infty$ for $r \in \mathbf{N}$, then $f^{(r)} \in L_{p}$ and

$$
\left\|f^{(r)}\right\|_{p} \leq C_{1}(r) \sum_{k=1}^{\infty} k^{r-1} E_{k}(f)_{p} .
$$

For $p=\infty$ this fact was proved by Bernstein in [Be1], for other $p$ we refer to [p. 209, De-Lo] and [Ch. 5,6, Ti]. As a corollary (see [Steč]) we have the following inequality

$$
E_{n}\left(f^{(r)}\right)_{p} \leq C_{2}(r)\left(n^{r} E_{n}(f)_{p}+\sum_{k=n+1}^{\infty} k^{r-1} E_{k}(f)_{p}\right) .
$$

2000 Mathematics Subject Classification: Primary 46E35; Secondary 41A50, 26A33.

Key words and phrases: embedding theorems, best approximation, $(\lambda, \beta)$-derivative.

This work was supported by the Russian Foundation for Fundamental Research (grant no. 06-01-00268) and the Leading Scientific Schools (grant NSH-4681.2006.1). The second author acknowledges financial support from the European Commission (Contract MIF1-CT-2004-509465). The paper was partially written while the authors were staying at the Centre de Recerca Matematica (CRM) in Barcelona, Spain. They are grateful to the staff of CRM for their hospitality.

The paper is in final form and no version of it will be published elsewhere. 
On the other hand, one can write the inverse inequality (see [p. 206, De-Lo]):

$$
n^{r} E_{n}(f)_{p} \leq C_{3}(r) E_{n}\left(f^{(r)}\right)_{p} .
$$

Thus, for $\alpha \in(0,1)$ and $\varepsilon=\left\{\varepsilon_{n}=n^{-(r+\alpha)}\right\}, \delta=\left\{\delta_{n}=n^{-\alpha}\right\}$ the following function classes coincide:

$$
\begin{aligned}
E_{p}[\varepsilon] & =\left\{f \in L_{p}: E_{n}(f)_{p}=O\left[\varepsilon_{n}\right]\right\}, \\
W_{p}^{r} E[\delta] & =\left\{f \in L_{p}: f^{(r)} \in L_{p}, E_{n}\left(f^{(r)}\right)_{p}=O\left[\delta_{n}\right]\right\} .
\end{aligned}
$$

We shall obtain necessary and sufficient conditions for embedding theorems of some function classes which are more general than (2) and (3). We shall use the concept of a $(\lambda, \beta)$-derivative, which allows us to consider $f^{(r)}$ as well as $\widetilde{f}^{(r)}$.

As an $r$-th derivative we shall consider the fractional derivative in the sense of Weyl. We would like to mention earlier papers [Ha-Li], [Kr], [Mu], [Og] in which this concept was used to examine the question mentioned above. Also we mention papers [Be1], [Ch-Zh], [Ha-Sh], [Mo], [Steč] where the results were obtained in the necessity part.

The paper is organized in the following way. Section 2 contains some definitions and preliminaries. In section 3, we present our main theorems. Section 4 contains lemmas. Sections 5 and 6 include the proofs of the main results for the cases $1<p<\infty$ and $p=1, \infty$, respectively.

Finally, we mention the paper by Stepanets [Step] where the analogues of inequality (1) for $(\lambda, \beta)$-derivatives were obtained. Our theorems are stronger for the case of $1<$ $p<\infty$.

2. Definition and notation. Let $L_{p}=L_{p}[0,2 \pi](1 \leq p<\infty)$ be the space of $2 \pi$ periodic functions for which $|f|^{p}$ is integrable, and $L_{\infty} \equiv C[0,2 \pi]$ be the space of $2 \pi$ periodic continuous functions with $\|f\|_{\infty}=\max \{|f(x)|, 0 \leq x \leq 2 \pi\}$.

Let a function $f \in L_{1}$ have the Fourier series

$$
f(x) \sim \sigma(f):=\frac{a_{0}(f)}{2}+\sum_{\nu=1}^{\infty}\left(a_{\nu}(f) \cos \nu x+b_{\nu}(f) \sin \nu x\right) \equiv \sum_{\nu=0}^{\infty} A_{\nu}(f, x) .
$$

By the transformed Fourier series of (4) we mean the series

$$
\sigma(f, \lambda, \beta):=\sum_{\nu=1}^{\infty} \lambda_{\nu}\left[a_{\nu} \cos \left(\nu x+\frac{\pi \beta}{2}\right)+b_{\nu} \sin \left(\nu x+\frac{\pi \beta}{2}\right)\right]
$$

where $\beta \in \mathbf{R}$ and $\lambda=\left\{\lambda_{n}\right\}$ is a given sequence of positive numbers. The sequence $\lambda=\left\{\lambda_{n}\right\}$ satisfies the $\triangle_{2}$-condition if $\lambda_{2 n} \leq C \lambda_{n}$ for all $n \in \mathbf{N}$. For $\lambda=\left\{\lambda_{n}\right\}_{n \in \mathbf{N}}$ we define $\triangle \lambda_{n}:=\lambda_{n}-\lambda_{n+1} ; \triangle^{2} \lambda_{n}:=\triangle\left(\triangle \lambda_{n}\right)$.

Let $S_{n}(f)$ denote the $n$-th partial sum of $(4), V_{n}(f)$ denote the de la Vallée-Poussin sum and $K_{n}(x)$ be the Fejér kernel, i.e.

$$
S_{n}(f)=\sum_{\nu=0}^{n} A_{\nu}(x), \quad V_{n}(f)=\frac{1}{n} \sum_{\nu=n}^{2 n-1} S_{\nu}(f), \quad K_{n}(x)=\frac{1}{n+1} \sum_{\nu=0}^{n}\left(\frac{1}{2}+\sum_{m=1}^{\nu} \cos m x\right) .
$$

Let $E_{n}(f)_{p}$ be the best approximation of a function $f$ by trigonometric polynomials of 
order no more than $n$, i.e.

$$
E_{n}(f)_{p}=\inf _{\alpha_{k}, \beta_{k} \in \mathbf{R}}\left\|f(x)-\sum_{k=0}^{n}\left(\alpha_{k} \cos k x+\beta_{k} \sin k x\right)\right\|_{p}
$$

Let $\Phi$ be the class of all decreasing null-sequences. For $\beta \in \mathbf{R}$ and $\lambda=\left\{\lambda_{n}>0\right\}$ we define the following function class:

$$
W_{p}^{\lambda, \beta}=\left\{f \in L_{p}: \exists g \in L_{p}, g(x) \sim \sigma(f, \lambda, \beta)\right\} .
$$

We call the function $g(x) \sim \sigma(f, \lambda, \beta)$ the $(\lambda, \beta)$-derivative of the function $f(x)$ and denote it by $f^{(\lambda, \beta)}(x)$. Also, we define for $\varepsilon \in \Phi$

$$
W_{p}^{\lambda, \beta} E[\varepsilon]=\left\{f \in W_{p}^{\lambda, \beta}: E_{n}\left(f^{(\lambda, \beta)}\right)_{p}=O\left[\varepsilon_{n}\right]\right\}
$$

In the case $\lambda_{n} \equiv 1$ and $\beta=0$ the class $W_{p}^{\lambda, \beta} E[\varepsilon]$ coincides with the class $E_{p}[\varepsilon]$ (see (2)).

It is clear that if $\lambda_{n}=n^{r}, r>0, \beta=r$, then the class $W_{p}^{\lambda, \beta} E[\varepsilon]$ coincides with the class $W_{p}^{r} E[\varepsilon]$ (see (3) where $f^{(r)}$ denotes a fractional derivative in the Weyl sense) and if $\lambda_{n}=n^{r}, r>0, \beta=r+1$, then the class $W_{p}^{\lambda, \beta} E[\varepsilon]$ coincides with the class

$$
\widetilde{W}_{p}^{r} E[\varepsilon]:=\left\{f \in L_{p}: \widetilde{f}^{(r)} \in L_{p}, E_{n}\left(\widetilde{f}^{(r)}\right)_{p}=O\left[\varepsilon_{n}\right]\right\}
$$

Here and further, $\widetilde{f}$ is a conjugate function to $f$.

By $C(s, t, \ldots)$ we denote the positive constants that are dependent only on $s, t, \cdots$ and may be different in different formulas.

\section{Main results}

TheOrem 1. Let $1<p<\infty, \theta=\min (2, p), \beta \in \mathbf{R}$, and $\lambda=\left\{\lambda_{n}\right\}$ be a non-decreasing sequence of positive numbers satisfying the $\triangle_{2}$-condition. Let $\varepsilon=\left\{\varepsilon_{n}\right\}, \omega=\left\{\omega_{n}\right\} \in \Phi$. Then

$$
\begin{aligned}
& E_{p}[\varepsilon] \subset W_{p}^{\lambda, \beta} \Leftrightarrow \sum_{n=1}^{\infty}\left(\lambda_{n+1}^{\theta}-\lambda_{n}^{\theta}\right) \varepsilon_{n}^{\theta}<\infty, \\
& E_{p}[\varepsilon] \subset W_{p}^{\lambda, \beta} E[\omega] \Leftrightarrow\left\{\sum_{\nu=n+1}^{\infty}\left(\lambda_{\nu+1}^{\theta}-\lambda_{\nu}^{\theta}\right) \varepsilon_{\nu}^{\theta}\right\}^{\frac{1}{\theta}}+\lambda_{n} \varepsilon_{n}=O\left[\omega_{n}\right], \\
& W_{p}^{\lambda, \beta} \subset E_{p}[\varepsilon] \Leftrightarrow \frac{1}{\lambda_{n}}=O\left[\varepsilon_{n}\right], \\
& W_{p}^{\lambda, \beta} E[\omega] \subset E_{p}[\varepsilon] \Leftrightarrow \frac{\omega_{n}}{\lambda_{n}}=O\left[\varepsilon_{n}\right] .
\end{aligned}
$$

THEOREM 2. Let $p=1, \infty, \beta \in \mathbf{R}$, and $\lambda=\left\{\lambda_{n}\right\}$ be a non-decreasing sequence of positive numbers satisfying the $\triangle_{2}$-condition. Let $\varepsilon=\left\{\varepsilon_{n}\right\}, \omega=\left\{\omega_{n}\right\} \in \Phi$. 
A. If $\triangle \lambda_{n} \leq C \triangle \lambda_{2 n}$ and $\triangle^{2} \lambda_{n} \geq 0($ or $\leq 0)$, then

$$
\begin{aligned}
& E_{p}[\varepsilon] \subset W_{p}^{\lambda, \beta} \Leftrightarrow \mid \cos \frac{\beta \pi}{2} \mid \sum_{n=1}^{\infty}\left(\lambda_{n+1}-\lambda_{n}\right) \varepsilon_{n} \\
&+\left|\sin \frac{\beta \pi}{2}\right| \sum_{n=1}^{\infty} \lambda_{n} \frac{\varepsilon_{n}}{n}<\infty, \\
& E_{p}[\varepsilon] \subset W_{p}^{\lambda, \beta} E[\omega] \Leftrightarrow\left|\cos \frac{\beta \pi}{2}\right| \sum_{\nu=n+1}^{\infty}\left(\lambda_{\nu+1}-\lambda_{\nu}\right) \varepsilon_{\nu}+\lambda_{n} \varepsilon_{n} \\
&+\left|\sin \frac{\beta \pi}{2}\right| \sum_{\nu=n+1}^{\infty} \lambda_{\nu} \frac{\varepsilon_{\nu}}{\nu}=O\left[\omega_{n}\right] .
\end{aligned}
$$

B. If for $\beta=2 k, k \in \mathbf{Z}$ the condition $\triangle^{2}\left(1 / \lambda_{n}\right) \geq 0$ holds, and for $\beta \neq 2 k, k \in \mathbf{Z}$ the conditions $\triangle^{2}\left(1 / \lambda_{n}\right) \geq 0$ and $\sum_{\nu=n+1}^{\infty} \frac{1}{\nu \lambda_{\nu}} \leq \frac{C}{\lambda_{n}}$ are fulfilled, then

$$
\begin{array}{r}
W_{p}^{\lambda, \beta} \subset E_{p}[\varepsilon] \Leftrightarrow \frac{1}{\lambda_{n}}=O\left[\varepsilon_{n}\right], \\
W_{p}^{\lambda, \beta} E[\omega] \subset E_{p}[\varepsilon] \Leftrightarrow \frac{\omega_{n}}{\lambda_{n}}=O\left[\varepsilon_{n}\right] .
\end{array}
$$

One can draw many conclusions from the inequalities which we use in the proofs of Theorems 1 and 2. The simplest ones are

Corollary 1. Let $1<p<\infty, \theta=\min (2, p)$, and $r>0, A \geq 0$. If for $f \in L_{p}$ the series

$$
\sum_{k=1}^{\infty} k^{r \theta-1} \ln ^{A \theta} k E_{k}^{\theta}(f)_{p}
$$

converges, then there exists $f^{(\lambda, \beta)} \in L_{p}$ with $\lambda=\left\{n^{r} \ln ^{A} n\right\}$ and $\beta \in \mathbf{R}$, and

$$
E_{n}\left(f^{(\lambda, \beta)}\right)_{p} \leq C(r, A, p)\left(n^{r} \ln ^{A} n E_{n}(f)_{p}+\left\{\sum_{k=n+1}^{\infty} k^{r \theta-1} \ln ^{A \theta} k E_{k}^{\theta}(f)_{p}\right\}^{\frac{1}{\theta}}\right) .
$$

Corollary 2. Let $p=1, \infty$, and $r>0, A \geq 0$. If for $f \in L_{p}$ the series

$$
\sum_{k=1}^{\infty} k^{r-1} \ln ^{A} k E_{k}(f)_{p}
$$

converges, then there exist $f^{(\lambda, \beta)}, \tilde{f}^{(\lambda, \beta)} \in L_{p}$ with $\lambda=\left\{n^{r} \ln ^{A} n\right\}$ and $\beta \in \mathbf{R}$, and

$$
E_{n}\left(f^{(\lambda, \beta)}\right)_{p}+E_{n}\left(\tilde{f}^{(\lambda, \beta)}\right)_{p} \leq C(r, A)\left(n^{r} \ln ^{A} n E_{n}(f)_{p}+\sum_{k=n+1}^{\infty} k^{r-1} \ln ^{A} k E_{k}(f)_{p}\right) .
$$

Corollary 3. Let $1 \leq p \leq \infty$, and $r>0, A \geq 0$. If for $f \in L_{p}$ there exist $f^{(\lambda, \beta)}, \tilde{f}^{(\lambda, \beta)} \in L_{p}$ with $\lambda=\left\{n^{r} \ln ^{A} n\right\}$ and $\beta \in \mathbf{R}$, then

$$
\begin{aligned}
& n^{r} \ln ^{A} n E_{n}(f)_{p} \leq C(r, A, p) E_{n}\left(f^{(\lambda, \beta)}\right)_{p} . \\
& n^{r} \ln ^{A} n E_{n}(f)_{p} \leq C(r, A, p) E_{n}\left(\tilde{f}^{(\lambda, \beta)}\right)_{p} .
\end{aligned}
$$

We note that if $\lambda=\left\{n^{r} \ln ^{A} n\right\}$, then $f^{(\lambda, \beta)}$ is a fractional-logarithmic derivative of $f$ (see, for example, $[\mathrm{Ku}]$ ). 


\section{Auxiliary results}

Lemma 1 ([V. 1, p. 215, Zy]). Let $f(x)$ have the Fourier series $\sum_{\nu=1}^{\infty}\left(a_{\nu} \cos n_{\nu} x+\right.$ $\left.b_{\nu} \sin n_{\nu} x\right)$, where $n_{\nu+1} / n_{\nu} \geq q>1$ and $\sum_{\nu=1}^{\infty}\left(a_{\nu}^{2}+b_{\nu}^{2}\right)<\infty$. Then for $1 \leq p<\infty$

$$
C_{1}(p, q)\left\{\sum_{\nu=1}^{\infty}\left(a_{\nu}^{2}+b_{\nu}^{2}\right)\right\}^{\frac{1}{2}} \leq\|f\|_{p} \leq C_{2}(p, q)\left\{\sum_{\nu=1}^{\infty}\left(a_{\nu}^{2}+b_{\nu}^{2}\right)\right\}^{\frac{1}{2}}
$$

Lemma 2 ([Vol. 2, p. 269, Ba2]). Let $f(x) \in L_{\infty}$ have the Fourier series $\sum_{\nu=1}^{\infty}\left(a_{\nu} \cos n_{\nu} x\right.$ $\left.+b_{\nu} \sin n_{\nu} x\right)$, where $a_{\nu}, b_{\nu} \geq 0$ and $n_{\nu+1} / n_{\nu} \geq q>1$. Then

$$
C_{1}(q) \sum_{n_{\nu}>n}\left(a_{\nu}+b_{\nu}\right) \leq E_{n}(f)_{\infty} \leq C_{2}(q) \sum_{n_{\nu}>n}\left(a_{\nu}+b_{\nu}\right)
$$

Lemma 3 ([Steč $])$. Let $f(x) \in L_{p}, p=1, \infty$, with $\sum_{n=1}^{\infty} n^{-1} E_{n}(f)_{p}<\infty$. Then $\tilde{f}(x) \in L_{p}$ and

$$
E_{n}(\tilde{f})_{p} \leq C\left(E_{n}(f)_{p}+\sum_{k=n+1}^{\infty} k^{-1} E_{k}(f)_{p}\right) \quad(k \in \mathbf{N}) .
$$

Lemma 4 ([V. 1, p. 182, Zy]). Let $\varepsilon_{n} \downarrow 0$. The condition $\nu \varepsilon_{\nu} \rightarrow 0$ is both necessary and sufficient for $\sum_{\nu=1}^{\infty} \varepsilon_{\nu} \sin \nu x$ to be the Fourier series of a continuous function.

Lemma 5 ([Te]). Let $f(x) \in L_{1}$ have a Fourier series (4). Then

$$
E_{n}(f)_{1} \geq C \sum_{\nu=n+1}^{\infty} \frac{b_{\nu}}{\nu} .
$$

Lemma 6. Let $1 \leq p \leq \infty$ and $E_{p}[\varepsilon] \subset W_{p}^{\lambda, \beta} E[\omega]$. Then

$$
\lambda_{n} \varepsilon_{n}=O\left(\omega_{n}\right) \quad n \rightarrow \infty .
$$

Proof. Suppose that (15) does not hold. Then there exists a sequence $\left\{m_{n}\right\}$ such that $\lambda_{m_{n}} \varepsilon_{m_{n}} \geq C_{n} \omega_{m_{n}}$ and $C_{n} \uparrow \infty$ as $n \rightarrow \infty$. One can also choose a subsequence $\left\{m_{n_{k}}\right\}$ such that

$$
\frac{m_{n_{k+1}}}{m_{n_{k}}} \geq 2, \quad \varepsilon_{m_{n_{k}}} \geq \frac{1}{2} \varepsilon_{m_{n_{k}}}+\varepsilon_{m_{n_{k+1}}} \quad \text { and } \quad \lambda_{m_{n_{k}}} \varepsilon_{m_{n_{k}}} \geq C_{n_{k}} \omega_{m_{n_{k}}} .
$$

Let us consider the case $1 \leq p<\infty$. We consider the series

$$
\sum_{k=0}^{\infty}\left(\varepsilon_{m_{n_{k}}}^{2}-\varepsilon_{m_{n_{k+1}}}^{2}\right)^{\frac{1}{2}} \cos \left(\left(m_{n_{k}}+1\right) x-\frac{\pi \beta}{2}\right) .
$$

Since

$$
\sum_{k=0}^{\infty}\left(\varepsilon_{m_{n_{k}}}^{2}-\varepsilon_{m_{n_{k+1}}}^{2}\right)=\varepsilon_{m_{n_{0}}}^{2}
$$

by Lemma 1 , the series (16) is the Fourier series of a function $f_{0}(x) \in L_{p}$ and $E_{s}\left(f_{0}\right)_{p} \leq$ $C \varepsilon_{s}$, i.e. $f_{0} \in E[\varepsilon]$. Then $f_{0} \in W_{p}^{\lambda, \beta} E[\omega]$. On the other hand,

$$
\begin{aligned}
& E_{m_{n_{k}}}\left(f_{0}^{(\lambda, \beta)}\right)_{p} \geq C \lambda_{m_{n_{k}}+1}\left(\varepsilon_{m_{n_{k}}}^{2}-\varepsilon_{m_{n_{k+1}}}^{2}\right)^{\frac{1}{2}} \\
& =C \lambda_{m_{n_{k}}+1}\left[\left(\varepsilon_{m_{n_{k}}}-\varepsilon_{m_{n_{k+1}}}\right)\left(\varepsilon_{m_{n_{k}}}+\varepsilon_{m_{n_{k+1}}}\right)\right]^{\frac{1}{2}} \geq C \lambda_{m_{n_{k}}} \varepsilon_{m_{n_{k}}} \geq C_{n_{k}} \omega_{m_{n_{k}}} .
\end{aligned}
$$

Thus, $f_{0} \notin W_{p}^{\lambda, \beta} E_{p}[\omega]$. This contradiction implies (15). 
Let $p=\infty$. Let us consider the series

$$
\sum_{k=0}^{\infty}\left(\varepsilon_{m_{n_{k}}}-\varepsilon_{m_{n_{k+1}}}\right) \cos \left(\left(m_{n_{k}}+1\right) x-\frac{\pi \beta}{2}\right) .
$$

By Lemma 2, there exists $f_{1} \in L_{p}$ with Fourier series (17) and $E_{s}\left(f_{1}\right)_{p} \leq C \varepsilon_{s}$, i.e. $f_{1} \in E_{p}[\varepsilon] \subset W_{p}^{\lambda, \beta} E[\omega]$. On the other hand,

$$
E_{m_{n_{k}}}\left(f_{1}^{(\lambda, \beta)}\right)_{p} \geq C \lambda_{m_{n_{k}}+1}\left(\varepsilon_{m_{n_{k}}}-\varepsilon_{m_{n_{k+1}}}\right) \geq C \lambda_{m_{n_{k}}} \varepsilon_{m_{n_{k}}} \geq C C_{n_{k}} \omega_{m_{n_{k}}},
$$

i.e. $f_{1} \notin W_{p}^{\lambda, \beta} E[\omega]$.

Lemma 7 ([Si-Ti] ). Let $p=1, \infty$ and $\left\{\lambda_{n}\right\}$ be a monotonic concave (or convex) sequence. Let

$$
\begin{aligned}
T_{n}(x) & =\sum_{\nu=0}^{n} a_{\nu} \cos \nu x+b_{\nu} \sin \nu x, \\
T_{n}(\lambda, x) & =\sum_{\nu=0}^{n} \lambda_{\nu}\left(a_{\nu} \cos \nu x+b_{\nu} \sin \nu x\right) .
\end{aligned}
$$

Then for $M>N \geq 0$ one has

$$
\left\|T_{M}(\lambda, x)-T_{N}(\lambda, x)\right\|_{p} \leq \mu(M, N)\left\|T_{M}(x)-T_{N}(x)\right\|_{p},
$$

where

$$
\mu(M, N)= \begin{cases}2 M\left(\lambda_{M}-\lambda_{M-1}\right)+\lambda_{N+1}-(N+1)\left(\lambda_{N+2}-\lambda_{N+1}\right), & \text { if } \lambda_{n} \uparrow, \triangle^{2} \lambda_{n} \geq 0 ; \\ 2 \lambda_{M}+(N+1)\left(\lambda_{N+2}-\lambda_{N+1}\right)-\lambda_{N+1}, & \text { if } \lambda_{n} \uparrow, \triangle^{2} \lambda_{n} \leq 0 ; \\ (N+1)\left(\lambda_{N+1}-\lambda_{N+2}\right)+\lambda_{N+1}, & \text { if } \lambda_{n} \downarrow, \triangle^{2} \lambda_{n} \geq 0 .\end{cases}
$$

Lemma 8. Let $p=1, \infty$. Set

$$
T_{2^{n}, 2^{n+1}}(x)=\sum_{\nu=2^{n}}^{2^{n+1}}\left(c_{\nu} \cos \nu x+d_{\nu} \sin \nu x\right) .
$$

Then

$$
C_{1}\left\|\widetilde{T}_{2^{n}, 2^{n+1}}(\cdot)\right\|_{p} \leq\left\|T_{2^{n}, 2^{n+1}}(\cdot)\right\|_{p} \leq C_{2}\left\|\widetilde{T}_{2^{n}, 2^{n+1}}(\cdot)\right\|_{p} .
$$

Proof. We rewrite $T_{2^{n}, 2^{n+1}}(x)$ in the following way

$$
T_{2^{n}, 2^{n+1}}(x)=\sum_{\nu=2^{n}}^{2^{n+1}} \frac{1}{\nu}\left(\nu c_{\nu} \cos \nu x+\nu d_{\nu} \sin \nu x\right) .
$$

Applying Lemma 7 and the Bernstein inequality we have

$$
\begin{aligned}
\left\|T_{2^{n}, 2^{n+1}}(\cdot)\right\|_{p} & \leq C \frac{1}{2^{n}}\left\|\sum_{\nu=2^{n}}^{2^{n+1}}\left(\nu c_{\nu} \cos \nu x+\nu d_{\nu} \sin \nu x\right)\right\|_{p} \\
& =C \frac{1}{2^{n}}\left\|\left(\sum_{\nu=2^{n}}^{2^{n+1}}-d_{\nu} \cos \nu x+c_{\nu} \sin \nu x\right)^{\prime}\right\|_{p} \\
& \leq C\left\|\widetilde{T}_{2^{n}, 2^{n+1}}(\cdot)\right\|_{p} .
\end{aligned}
$$

The same reasoning for $\widetilde{T}_{2^{n}, 2^{n+1}}(x)$ implies the correctness of the left-hand side of (18). 
5. Proof of Theorem 1. We divide the proof of Theorem 1 into two parts.

\subsection{Proof of sufficiency}

STEP 1. Let us prove the sufficiency part in (7). First, if $\lambda_{n} \equiv 1$, the Riesz inequality ([V. 1, p. 253, Zy]) $\|\tilde{f}\|_{p} \leq C(p)\|f\|_{p}$ implies

$$
\left\|f^{(\lambda, \beta)}\right\|_{p} \leq C(p, \beta)\|f\|_{p}
$$

Let the series in the right part of (7) be convergent and $f \in E_{p}[\varepsilon]$. We use the following representation

$$
\lambda_{2^{n}}^{\theta}=\lambda_{1}^{\theta}+\sum_{\nu=2}^{n+1}\left(\lambda_{2^{\nu-1}}^{\theta}-\lambda_{2^{\nu-2}}^{\theta}\right) .
$$

Applying the Minkowski's inequality we get (here and further $\triangle_{1}:=A_{1}(f, x), \triangle_{n+2}:=$ $\sum_{\nu=2^{n}+1}^{2^{n+1}} A_{\nu}(f, x), n=0,1,2, \ldots$, where $A_{\nu}(f, x)$ is from $\left.(4)\right)$

$$
\begin{aligned}
I_{1} & :=\left\{\int_{0}^{2 \pi}\left[\sum_{n=1}^{\infty} \lambda_{2^{n-1}}^{2} \triangle_{n}^{2}\right]^{\frac{p}{2}} d x\right\}^{\frac{1}{p}} \leq C(\lambda)\left(\int_{0}^{2 \pi}\left\{\lambda_{1}^{2} \triangle_{1}^{2}+\lambda_{2}^{2} \triangle_{2}^{2}+\sum_{n=3}^{\infty} \lambda_{2^{n-2}}^{2} \triangle_{n}^{2}\right\}^{\frac{p}{2}} d x\right)^{\frac{1}{p}} \\
& \leq C(p, \lambda)\left(\int_{0}^{2 \pi}\left\{\lambda_{1}^{2} \triangle_{1}^{2}+\lambda_{2}^{2} \triangle_{2}^{2}+\sum_{n=3}^{\infty} \triangle_{n}^{2}\left[\lambda_{1}^{\theta}+\sum_{\nu=3}^{n}\left(\lambda_{2^{\nu-2}}^{\theta}-\lambda_{2^{\nu-3}}^{\theta}\right)\right]^{\frac{2}{\theta}}\right\}^{\frac{p}{2}} d x\right)^{\frac{1}{p}} \\
& \leq C(p, \lambda)\left(\lambda_{1}^{\theta}\left\{\int_{0}^{2 \pi}\left[\sum_{n=1}^{\infty} \triangle_{n}^{2}\right]^{\frac{p}{2}} d x\right\}^{\frac{\theta}{p}}+\sum_{s=3}^{\infty}\left(\lambda_{2^{s-2}}^{\theta}-\lambda_{2^{s-3}}^{\theta}\left\{\int_{0}^{2 \pi}\left[\sum_{n=s}^{\infty} \triangle_{n}^{2}\right]^{\frac{p}{2}} d x\right\}^{\frac{\theta}{p}}\right)^{\frac{1}{\theta}} .\right.
\end{aligned}
$$

By the Littlewood-Paley theorem ([V. II, p. 233, Zy]) and

$$
\left\|f-S_{n}(f)\right\|_{p} \leq C(p) E_{n}(f)_{p},
$$

we get

$$
I_{1} \leq C(p, \lambda)\left\{\lambda_{1}^{\theta} E_{0}^{\theta}(f)_{p}+\sum_{s=1}^{\infty}\left(\lambda_{2^{s}}^{\theta}-\lambda_{2^{s-1}}^{\theta}\right) E_{2^{s}}^{\theta}(f)_{p}\right\}^{\frac{1}{\theta}} .
$$

Since $f \in E_{p}[\varepsilon]$ we have $I_{1}<\infty$. Thus, by the Littlewood-Paley theorem, there exists a function $g \in L_{p}$ with Fourier series

$$
\sum_{n=1}^{\infty} \lambda_{2^{n-1}} \triangle_{n}
$$

and $\|g\|_{p} \leq C(p) I_{1}$. We rewrite series (21) in the form of $\sum_{n=1}^{\infty} \gamma_{n} A_{n}(f, x)$, where $\gamma_{i}:=$ $\lambda_{i}, i=1,2$ and $\gamma_{\nu}:=\lambda_{2^{n}}$ for $2^{n-1}+1 \leq \nu \leq 2^{n}(n=2,3, \cdots)$. Further, we write the series

$$
\sum_{n=1}^{\infty} \lambda_{n} A_{n}(f, x)=\sum_{n=1}^{\infty} \gamma_{n} \Lambda_{n} A_{n}(f, x),
$$

where $\Lambda_{1}:=\Lambda_{2}:=1, \Lambda_{\nu}:=\lambda_{\nu} / \gamma_{n}=\lambda_{\nu} / \lambda_{2^{n}}$ for $2^{n-1}+1 \leq \nu \leq 2^{n}(n=2,3, \cdots)$. The sequence $\left\{\Lambda_{n}\right\}$ satisfies the conditions of the Marcinkiewicz multiplier theorem ([V. II, p. 232, Zy]), i.e. the series (22) is the Fourier series of a function $f^{(\lambda, 0)} \in L_{p},\left\|f^{(\lambda, 0)}\right\|_{p} \leq$ $C(p, \lambda)\|g\|_{p}$. 
Using the properties of $\left\{\lambda_{n}\right\}$ and (19), we write

$$
\begin{aligned}
\left\|f^{(\lambda, \beta)}\right\|_{p} & \leq C(p, \lambda)\left\{\lambda_{1}^{\theta} E_{0}^{\theta}(f)_{p}+\sum_{s=1}^{\infty} E_{2^{s}}^{\theta}(f)_{p} \sum_{n=2^{s-1}}^{2^{s}-1}\left(\lambda_{n+1}^{\theta}-\lambda_{n}^{\theta}\right)\right\}^{\frac{1}{\theta}} \\
& \leq C(p, \lambda)\left\{\lambda_{1}^{\theta} E_{0}^{\theta}(f)_{p}+\sum_{n=1}^{\infty}\left(\lambda_{n+1}^{\theta}-\lambda_{n}^{\theta}\right) E_{n}^{\theta}(f)_{p}\right\}^{\frac{1}{\theta}} .
\end{aligned}
$$

Thus, the sufficiency in (7) has been proved.

STEP 2. Let the relation in the right-hand side of (8) hold, and $f \in E_{p}[\varepsilon]$. Let us prove $f \in W_{p}^{\lambda, \beta} E[\omega]$. We have

$$
E_{n}\left(f^{(\lambda, \beta)}\right)_{p} \leq\left\|f^{(\lambda, \beta)}-S_{n}\left(f^{(\lambda, \beta)}\right)\right\|_{p}=\left\|\left(f-S_{n}\right)^{(\lambda, \beta)}\right\|_{p} .
$$

Applying (23) for the function $f-S_{n}$ we get

$$
\begin{aligned}
& E_{n}\left(f^{(\lambda, \beta)}\right)_{p} \leq C(p, \lambda)\left\{\lambda_{1}^{\theta} E_{0}^{\theta}\left(f-S_{n}\right)_{p}+\sum_{m=1}^{\infty}\left(\lambda_{m+1}^{\theta}-\lambda_{m}^{\theta}\right) E_{m}^{\theta}\left(f-S_{n}\right)_{p}\right\}^{\frac{1}{\theta}} \\
& \leq C(p, \lambda)\left\{\lambda_{1}^{\theta} E_{n}^{\theta}(f)_{p}+E_{n}^{\theta}(f)_{p} \sum_{m=1}^{n}\left(\lambda_{m+1}^{\theta}-\lambda_{m}^{\theta}\right)+\sum_{m=n+1}^{\infty}\left(\lambda_{m+1}^{\theta}-\lambda_{m}^{\theta}\right) E_{m}^{\theta}(f)_{p}\right\}^{\frac{1}{\theta}} \\
& \leq C(p, \lambda)\left\{\lambda_{n}^{\theta} E_{n}^{\theta}(f)_{p}+\sum_{m=n+1}^{\infty}\left(\lambda_{m+1}^{\theta}-\lambda_{m}^{\theta}\right) E_{m}^{\theta}(f)_{p}\right\}^{\frac{1}{\theta}} \leq C(p, \lambda) \omega_{n} .
\end{aligned}
$$

This proves the sufficiency in (8).

STEP 3. Now we shall prove that the conditions $\frac{1}{\lambda_{n}}=O\left[\varepsilon_{n}\right]$ and $\frac{\omega_{n}}{\lambda_{n}}=O\left[\varepsilon_{n}\right]$ are sufficient for $W_{p}^{\lambda, \beta} \subset E_{p}[\varepsilon]$ and $W_{p}^{\lambda, \beta} E[\omega] \subset E_{p}[\varepsilon]$, respectively.

Let $f \in W_{p}^{\lambda, \beta}$. From the properties of the sequence $\left\{\lambda_{n}\right\}$, using the Littlewood-Paley and the Marcinkiewicz multiplier theorem, we get

$$
E_{n}(f)_{p} \leq\left\|f-S_{n}(f)\right\|_{p} \leq \frac{C(p, \lambda)}{\lambda_{n}}\left\|f^{(\lambda, \beta)}-S_{n}\left(f^{(\lambda, \beta)}\right)\right\|_{p} \leq \frac{C(p, \lambda)}{\lambda_{n}} E_{n}\left(f^{(\lambda, \beta)}\right)_{p} .
$$

If $\frac{1}{\lambda_{n}}=O\left[\varepsilon_{n}\right]$, then $E_{n}(f)_{p} \leq \frac{C}{\lambda_{n}}=O\left[\varepsilon_{n}\right]$, and if $\frac{\omega_{n}}{\lambda_{n}}=O\left[\varepsilon_{n}\right]$, then $E_{n}(f)_{p} \leq \frac{C}{\lambda_{n}} E_{n}\left(f^{(\lambda, \beta)}\right)_{p}$ $\leq C \frac{\omega_{n}}{\lambda_{n}}=O\left[\varepsilon_{n}\right]$, i.e. $f \in E_{p}[\varepsilon]$.

The proof of the sufficiency part in (9) and (10) is complete.

\subsection{Proof of necessity}

STEP 4. Let us prove the necessity part in (7). Let $E_{p}[\varepsilon] \subset W_{p}^{\lambda, \beta}$ but the series in (7) be divergent.

Let $2 \leq p<\infty$. We consider the series

$$
\sum_{\nu=0}^{\infty}\left(\varepsilon_{2^{\nu}-1}^{2}-\varepsilon_{2^{\nu+1}-1}^{2}\right)^{\frac{1}{2}} \cos 2^{\nu} x .
$$

Since $\sum_{\nu=0}^{\infty}\left(\varepsilon_{2^{\nu}-1}^{2}-\varepsilon_{2^{\nu+1}-1}^{2}\right)=\varepsilon_{0}^{2}$, by Lemma 1 , the series (24) is the Fourier series of a function $f_{2}(x) \in L_{p}$. 
Let $2^{\nu}-1 \leq n<2^{\nu+1}-1$. Then $E_{n}\left(f_{2}\right)_{p} \leq\left\|f_{2}-S_{n}\left(f_{2}\right)\right\|_{p} \leq C \varepsilon_{n}$, i.e. $f_{2} \in E_{p}[\varepsilon] \subset$ $W_{p}^{\lambda, \beta}$. On the other hand,

$$
\begin{aligned}
\left\|f_{2}^{(\lambda, \beta)}\right\|_{p} & \geq C\left\{\sum_{\nu=0}^{\infty}\left(\varepsilon_{2^{\nu}-1}^{2}-\varepsilon_{2^{\nu+1}-1}^{2}\right) \lambda_{2^{\nu}}^{2}\right\}^{\frac{1}{2}} \\
& \geq C\left\{\sum_{\nu=0}^{\infty}\left(\varepsilon_{2^{\nu}-1}^{2}-\varepsilon_{2^{\nu+1}-1}^{2}\right)\left[\sum_{n=0}^{\nu}\left(\lambda_{2^{n+1}}^{2}-\lambda_{2^{n}}^{2}\right)+\lambda_{1}^{2}\right]\right\}^{\frac{1}{2}} \\
& =C\left\{\lambda_{1}^{2} \varepsilon_{0}^{2}+\sum_{n=0}^{\infty}\left(\lambda_{2^{n+1}}^{2}-\lambda_{2^{n}}^{2}\right) \varepsilon_{2^{n}-1}^{2}\right\}^{\frac{1}{2}} \\
& =C\left\{\lambda_{1}^{2} \varepsilon_{0}^{2}+\sum_{n=0}^{\infty} \sum_{\nu=2^{n}}^{2^{n+1}-1}\left(\lambda_{\nu+1}^{2}-\lambda_{\nu}^{2}\right) \varepsilon_{2^{n}-1}^{2}\right\}^{\frac{1}{2}} \\
& \geq C\left\{\lambda_{1}^{2} \varepsilon_{0}^{2}+\sum_{\nu=1}^{\infty}\left(\lambda_{\nu+1}^{2}-\lambda_{\nu}^{2}\right) \varepsilon_{\nu}^{2}\right\}^{\frac{1}{2}}=\infty
\end{aligned}
$$

This contradiction implies the convergence of series in (7).

Let now $1<p<2$. We shall consider the series

$$
\left(\varepsilon_{0}^{p}-\varepsilon_{1}^{p}\right)^{\frac{1}{p}} \cos x+\sum_{\nu=0}^{\infty} 2^{\nu\left(\frac{1}{p}-1\right)}\left(\varepsilon_{2^{\nu+1}-1}^{p}-\varepsilon_{2^{\nu+2}-1}^{p}\right)^{\frac{1}{p}} \sum_{\mu=2^{\nu+1}}^{2^{\nu+1}} \cos \mu x .
$$

By the Jensen inequality and

$$
C_{1}(p) 2^{\nu(p-1)} \leq\left\|\sum_{\mu=2^{\nu-1}+1}^{2^{\nu}} \cos \mu x\right\|_{p}^{p} \leq C_{2}(p) 2^{\nu(p-1)},
$$

we have

$$
\begin{aligned}
& \int_{0}^{2 \pi}\left\{\sum_{\nu=0}^{\infty}\left[\left(\varepsilon_{2^{\nu+1}-1}^{p}-\varepsilon_{2^{\nu+2}-1}^{p}\right)^{\frac{1}{p}} 2^{\nu\left(\frac{1}{p}-1\right)} \sum_{\mu=2^{\nu+1}}^{2^{\nu+1}} \cos \mu x\right]^{2}\right\}^{\frac{p}{2}} d x \\
\leq & \int_{0}^{2 \pi}\left[\sum_{\nu=0}^{\infty} 2^{\nu(1-p)}\left(\varepsilon_{2^{\nu+1}-1}^{p}-\varepsilon_{2^{\nu+2}-1}^{p}\right)\left|\sum_{\mu=2^{\nu-1}+1}^{2^{\nu}} \cos \mu x\right|^{p}\right] d x \leq C(p) \varepsilon_{1}^{p} .
\end{aligned}
$$

By the Littlewood-Paley theorem, there exists a function $f_{3} \in L_{p}$ with Fourier series (25). Let $n=2^{\nu}$. Then

$$
\begin{aligned}
\left\|f_{3}-S_{n}\left(f_{3}\right)\right\|_{p} & =\left\|\sum_{m=\nu}^{\infty} 2^{m\left(\frac{1}{p}-1\right)}\left(\varepsilon_{2^{m+1}-1}^{p}-\varepsilon_{2^{m+2}-1}^{p}\right)^{\frac{1}{p}} \sum_{\mu=2^{m}+1}^{2^{m+1}} \cos \mu x\right\|_{p} \\
& \leq\left\{\int_{0}^{2 \pi}\left[\sum_{m=\nu}^{\infty} 2^{m(1-p)}\left(\varepsilon_{2^{m+1}-1}^{p}-\varepsilon_{2^{m+2}-1}^{p}\right)\left|\sum_{\mu=2^{m}+1}^{2^{m+1}} \cos \mu x\right|^{p}\right] d x\right\}^{\frac{1}{p}} \\
& \leq C(p) \varepsilon_{2^{\nu+1}-1} \leq C(p) \varepsilon_{2^{\nu}} .
\end{aligned}
$$

Let $n=0$. Then $E_{0}\left(f_{3}\right)_{p} \leq C(p)\left(\varepsilon_{0}^{p}-\varepsilon_{1}^{p}+\varepsilon_{1}^{p}\right)^{\frac{1}{p}}=C(p) \varepsilon_{0}$. 
Let $2^{\nu}<n<2^{\nu+1}$. Then

$$
\begin{aligned}
\left\|f_{3}-S_{n}\left(f_{3}\right)\right\|_{p} & =\| 2^{\nu\left(\frac{1}{p}-1\right)}\left(\varepsilon_{2^{\nu+1}-1}^{p}-\varepsilon_{2^{\nu+2}-1}^{p}\right)^{\frac{1}{p}} \sum_{\mu=n+1}^{2^{\nu+1}} \cos \mu x \\
& +\sum_{m=\nu+1}^{\infty} 2^{m\left(\frac{1}{p}-1\right)}\left(\varepsilon_{2^{m+1}-1}^{p}-\varepsilon_{2^{m+2}-1}^{p}\right)^{\frac{1}{p}} \sum_{\mu=2^{m}+1}^{2^{m+1}} \cos \mu x \|_{p} \leq C(p) \varepsilon_{n} .
\end{aligned}
$$

Therefore, one has $f_{3} \in E_{p}[\varepsilon]$. By our assumption, this implies $f_{3}(x) \in W_{p}^{\lambda, \beta}$.

On the other hand, Paley's theorem on Fourier coefficients [V. 2, p. 121, Zy] implies

$$
\begin{aligned}
\left\|f_{3}^{(\lambda, \beta)}\right\|_{p}^{p} & \geq C(p)\left\{\left(\varepsilon_{0}^{p}-\varepsilon_{1}^{p}\right) \lambda_{1}^{p}+\sum_{\nu=0}^{\infty}\left(\varepsilon_{2^{\nu+1}-1}^{p}-\varepsilon_{2^{\nu+2}-1}^{p}\right) 2^{\nu(1-p)} \sum_{\mu=2^{\nu}+1}^{2^{\nu+1}} \lambda_{\mu}^{p} \mu^{p-2}\right\} \\
& \geq C(\lambda, p)\left\{\left(\varepsilon_{0}^{p}-\varepsilon_{1}^{p}\right) \lambda_{1}^{p}+\sum_{\nu=0}^{\infty}\left(\varepsilon_{2^{\nu}-1}^{p}-\varepsilon_{2^{\nu+1}-1}^{p}\right) \lambda_{2^{\nu}}^{p}\right\} \\
& \geq C(\lambda, p)\left\{\varepsilon_{0}^{p} \lambda_{1}^{p}+\sum_{\nu=1}^{\infty}\left(\lambda_{\nu+1}^{p}-\lambda_{\nu}^{p}\right) \varepsilon_{\nu}^{p}\right\}=\infty .
\end{aligned}
$$

This contradicts $f_{3}(x) \in W_{p}^{\lambda, \beta}$. The series in (7) converges.

STEP 5. Now we shall prove the necessity in (8). Let $2 \leq p<\infty$ and $2^{\nu} \leq n<2^{\nu+1}, \nu=$ $0,1,2, \ldots$ We consider

$$
\left(\varepsilon_{n}^{2}-\varepsilon_{2^{\nu+1}-1}^{2}\right)^{\frac{1}{2}} \cos (n+1) x+\sum_{\nu=0}^{\infty}\left(\varepsilon_{2^{\nu}-1}^{2}-\varepsilon_{2^{\nu+1}-1}^{2}\right)^{\frac{1}{2}} \cos 2^{\nu} x .
$$

Repeating the argument we used for series (24) we can see that the series (27) is the Fourier series of a function $f_{4, n} \in L_{p}$ and $f_{4, n} \in E_{p}[\varepsilon]$. Therefore, $f_{4, n} \in W_{p}^{\lambda, \beta} E[\omega]$.

Let us show that the positive constant $C_{1}$ in the inequality $E_{m}\left(f_{4, n}^{(\lambda, \beta)}\right)_{p} \leq C_{1} \omega_{m}$ $(m=0,1,2, \ldots)$ is independent of $m$ and $n$. Indeed, for the function

$$
f_{4, n}(x)=f_{2}(x)+\left(\varepsilon_{n}^{2}-\varepsilon_{2^{\nu+1}-1}^{2}\right)^{\frac{1}{2}} \cos (n+1) x
$$

one has

$$
\begin{aligned}
& E_{m}\left(f_{4, n}^{(\lambda, \beta)}\right)_{p} \leq\left\|f_{4, n}^{(\lambda, \beta)}-S_{m}\left(f_{4, n}^{(\lambda, \beta)}\right)\right\|_{p} \leq\left\|f_{2}^{(\lambda, \beta)}-S_{m}\left(f_{2}^{(\lambda, \beta)}\right)\right\|_{p} \\
+ & \left\|\left[\left(\varepsilon_{n}^{2}-\varepsilon_{2^{\nu+1}-1}^{2}\right)^{\frac{1}{2}} \cos (n+1) x\right]^{(\lambda, \beta)}-S_{m}\left(\left[\left(\varepsilon_{n}^{2}-\varepsilon_{2^{\nu+1}-1}^{2}\right)^{\frac{1}{2}} \cos (n+1) x\right]^{(\lambda, \beta)}\right)\right\|_{p} .
\end{aligned}
$$

Since $f_{2} \in E_{p}[\varepsilon] \subset W_{p}^{\lambda, \beta} E[\omega], E_{m}\left(f_{2}^{(\lambda, \beta)}\right)_{p}=O\left(\omega_{m}\right)$.

Then for $m \geq n+1: E_{m}\left(f_{4, n}^{(\lambda, \beta)}\right)_{p}=E_{m}\left(f_{2}^{(\lambda, \beta)}\right)_{p} \leq C\left(f_{2}, p, \lambda, \beta\right) \omega_{m}$ and for $0 \leq m \leq n$, $E_{m}\left(f_{4, n}^{(\lambda, \beta)}\right)_{p} \leq C\left(f_{2}, p, \lambda, \beta\right) \omega_{m}+C(p, \lambda) \lambda_{n} \varepsilon_{n}$. By Lemma 6 , we have

$$
E_{m}\left(f_{4, n}^{(\lambda, \beta)}\right)_{p} \leq C\left(f_{2}, p, \lambda, \beta\right) \omega_{m}+C(p, \lambda) \lambda_{m} \varepsilon_{m} \leq C\left(f_{2}, p, \lambda, \beta\right) \omega_{m} .
$$

Thus, $E_{m}\left(f_{4, n}^{(\lambda, \beta)}\right)_{p} \leq C_{1} \omega_{m}$ where $C_{1}$ does not depend on $n$ and $m$. 
On the other hand,

$$
\begin{aligned}
C \omega_{n} & \geq E_{n}\left(f_{4, n}^{(\lambda, \beta)}\right)_{p} \geq C(p, \lambda)\left[\left(\varepsilon_{n}^{2}-\varepsilon_{2^{\nu+1}-1}^{2}\right) \lambda_{n}^{2}+\sum_{m=\nu+1}^{\infty}\left(\varepsilon_{2^{m}-1}^{2}-\varepsilon_{2^{m+1}-1}^{2}\right) \lambda_{2^{m}}^{2}\right]^{\frac{1}{2}} \\
& \geq C(p, \lambda)\left[\left(\varepsilon_{n}^{2}-\varepsilon_{2^{\nu+1}-1}^{2}\right) \lambda_{n}^{2}+\lambda_{2^{\nu+1}-1}^{2} \varepsilon_{2^{\nu+1}-1}^{2}+\sum_{m=2^{\nu+1}}^{\infty}\left(\lambda_{m+1}^{2}-\lambda_{m}^{2}\right) \varepsilon_{m}^{2}\right]^{\frac{1}{2}} \\
& \geq C(p, \lambda)\left[\varepsilon_{n}^{2} \lambda_{n}^{2}+\left(\sum_{m=n+1}^{2^{\nu+1}-1}\left(\lambda_{m+1}^{2}-\lambda_{m}^{2}\right)+\lambda_{n+1}^{2}\right) \varepsilon_{n}^{2}+\sum_{m=2^{\nu+1}}^{\infty}\left(\lambda_{m+1}^{2}-\lambda_{m}^{2}\right) \varepsilon_{m}^{2}\right]^{\frac{1}{2}} \\
& \geq C(p, \lambda)\left[\varepsilon_{n}^{2} \lambda_{n}^{2}+\sum_{m=n+1}^{\infty}\left(\lambda_{m+1}^{2}-\lambda_{m}^{2}\right) \varepsilon_{m}^{2}\right]^{\frac{1}{2}} .
\end{aligned}
$$

Thus, the relation in the right-hand side of (8) holds.

Let $1<p<2$. For $n=0$ we consider the series (25). For $2^{m} \leq n<2^{m+1}, m=$ $0,1,2, \ldots$ we define

$$
\begin{array}{r}
\left(\varepsilon_{0}^{p}-\varepsilon_{1}^{p}\right)^{\frac{1}{p}} \cos x+\left(\sum_{\nu=0}^{m-1}+\sum_{\nu=m+1}^{\infty}\right) 2^{\nu\left(\frac{1}{p}-1\right)}\left(\varepsilon_{2^{\nu+1}-1}^{p}-\varepsilon_{2^{\nu+2}-1}^{p}\right)^{\frac{1}{p}} \sum_{\mu=2^{\nu}+1}^{2^{\nu+1}} \cos \mu x \\
+\left(\varepsilon_{n}^{p}-\varepsilon_{2^{m+2}-1}^{p}\right)^{\frac{1}{p}} \cos (n+1) x .
\end{array}
$$

Since

$$
\begin{aligned}
& \left\|\left(\varepsilon_{0}^{p}-\varepsilon_{1}^{p}\right)^{\frac{1}{p}} \cos x+\sum_{\nu=0}^{m-1} 2^{\nu\left(\frac{1}{p}-1\right)}\left(\varepsilon_{2^{\nu+1}-1}^{p}-\varepsilon_{2^{\nu+2}-1}^{p}\right)^{\frac{1}{p}} \sum_{\mu=2^{\nu}+1}^{2^{\nu+1}} \cos \mu x\right\|_{p}^{p} \\
& +\left(\varepsilon_{n}^{p}-\varepsilon_{2^{m+2}-1}^{p}\right)+\left\|\sum_{\nu=m+1}^{\infty} 2^{\nu\left(\frac{1}{p}-1\right)}\left(\varepsilon_{2^{\nu+1}-1}^{p}-\varepsilon_{2^{\nu+2}-1}^{p}\right)^{\frac{1}{p}} \sum_{\mu=2^{\nu+1}}^{2^{\nu+1}} \cos \mu x\right\|_{p}^{p} \\
\leq & C(p)\left(\varepsilon_{0}^{p}-\varepsilon_{1}^{p}+\varepsilon_{1}^{p}-\varepsilon_{2^{m+1}-1}^{p}+\varepsilon_{n}^{p}-\varepsilon_{2^{m+2}-1}^{p}+\varepsilon_{2^{m+2}-1}^{p}-\varepsilon_{2^{m+3}-1}^{p}+\cdots\right) \\
\leq & C(p) \varepsilon_{0}^{p},
\end{aligned}
$$

there exists a function $f_{5, n}(x) \in L_{p}$ with Fourier series (28). One can verify that $E_{0}\left(f_{5, n}\right)_{p} \leq C(p) \varepsilon_{0}, E_{k}\left(f_{5, n}\right)_{p} \leq C(p) \varepsilon_{k}$. Therefore, $f_{5, n} \in E_{p}[\varepsilon] \subset W_{p}^{\lambda, \beta} E[\omega]$.

Let us show that the positive constant $C_{2}$ in the inequality $E_{m}\left(f_{5, n}^{(\lambda, \beta)}\right)_{p} \leq C_{2} \omega_{m}$ $(m=0,1,2, \ldots)$ is independent of $m$ and $n$. We note

$$
\begin{array}{r}
f_{5, n}(x)=f_{3}(x)-\sum_{\mu=2^{m}+1}^{2^{m+1}} 2^{m\left(\frac{1}{p}-1\right)}\left(\varepsilon_{2^{m+1}-1}^{p}-\varepsilon_{2^{m+2}-1}^{p}\right)^{\frac{1}{p}} \cos \mu x \\
+\left(\varepsilon_{n}^{p}-\varepsilon_{2^{m+2}-1}^{p}\right)^{\frac{1}{p}} \cos (n+1) x .
\end{array}
$$

If $n=0,1$, then $E_{k}\left(f_{5, n}^{(\lambda, \beta)}\right)_{p}=E_{k}\left(f_{3}^{(\lambda, \beta)}\right)_{p} \leq C\left(f_{3}, p, \lambda, \beta\right) \omega_{k}$. If $n=2,3, \ldots$, and 
$2^{m} \leq n<2^{m+1}$, then for $k=0,1, \ldots, n$

$$
\begin{aligned}
E_{k}\left(f_{5, n}^{(\lambda, \beta)}\right)_{p} \leq & \left\|f_{5, n}^{(\lambda, \beta)}-S_{k}\left(f_{5, n}^{(\lambda, \beta)}\right)\right\|_{p} \leq\left\|f_{3}^{(\lambda, \beta)}-S_{k}\left(f_{3}^{(\lambda, \beta)}\right)\right\|_{p} \\
& +\left\|\left(\sum_{\mu=2^{m}+1}^{2^{m+1}} 2^{m\left(\frac{1}{p}-1\right)}\left(\varepsilon_{2^{m+1}-1}^{p}-\varepsilon_{2^{m+2}-1}^{p}\right)^{\frac{1}{p}} \cos \mu x\right)^{(\lambda, \beta)}\right\|_{p} \\
& +\left\|\left(\left(\varepsilon_{n}^{p}-\varepsilon_{2^{m+2}-1}^{p}\right)^{\frac{1}{p}} \cos (n+1) x\right)^{(\lambda, \beta)}\right\|_{p} .
\end{aligned}
$$

By Lemma 6, using $f_{3} \in W^{\lambda, \beta} E[\omega]$, we have $E_{k}\left(f_{5, n}^{(\lambda, \beta)}\right)_{p} \leq C\left(f_{3}, p, \lambda, \beta\right) \omega_{k}$ for $k=$ $0,1,2, \ldots, n$. For $k>n$ we write $E_{k}\left(f_{5, n}^{(\lambda, \beta)}\right)_{p} \leq\left\|f_{3}^{(\lambda, \beta)}-S_{k}\left(f_{3}^{(\lambda, \beta)}\right)\right\|_{p} \leq C\left(f_{3}, p, \lambda, \beta\right) \omega_{k}$.

Thus, $E_{k}\left(f_{5, n}^{(\lambda, \beta)}\right)_{p} \leq C_{2} \omega_{k}$ for any $k$ and $C_{2}$ is independent of $m$ and $n$. On the other hand,

$$
\begin{aligned}
& C_{2} \omega_{n} \geq E_{n}\left(f_{5, n}^{(\lambda, \beta)}\right)_{p} \geq C(p)\left\|f_{5, n}^{(\lambda, \beta)}-S_{k}\left(f_{5, n}^{(\lambda, \beta)}\right)\right\|_{p} \\
& \geq C(p)\left(\int _ { 0 } ^ { 2 \pi } \left\{\left\langle\left[\left(\varepsilon_{n}^{p}-\varepsilon_{2^{m+2}-1}^{p}\right)^{\frac{1}{p}} \cos (n+1) x\right]^{(\lambda, \beta)}\right\rangle^{2}\right.\right. \\
& \left.\left.+\sum_{\nu=m+1}^{\infty}\left\langle\left[\left(\varepsilon_{2^{\nu+1}-1}^{p}-\varepsilon_{2^{\nu+2}-1}^{p}\right)^{\frac{1}{p}} 2^{\nu\left(\frac{1}{p}-1\right)} \sum_{\mu=2^{\nu}+1}^{2^{\nu+1}} \cos \mu x\right]^{(\lambda, \beta)}\right\rangle^{2}\right\}^{\frac{p}{2}} d x\right)^{\frac{1}{p}} \\
& \geq C(p)\left\{\left[\left(\varepsilon_{n}^{p}-\varepsilon_{2^{m+2}-1}^{p}\right) \lambda_{n}^{p} \int_{0}^{2 \pi}|\cos (n+1) x|^{p} d x\right]^{\frac{1}{p}}\right. \\
& \left.+\left[\int_{0}^{2 \pi}\left(\sum_{\nu=m+1}^{\infty}\left\langle\left[\left(\varepsilon_{2^{\nu+1}-1}^{p}-\varepsilon_{2^{\nu+2}-1}^{p}\right)^{\frac{1}{p}} 2^{\nu\left(\frac{1}{p}-1\right)} \sum_{\mu=2^{\nu}+1}^{2^{\nu+1}} \cos \mu x\right]^{(\lambda, \beta)}\right\rangle^{2}\right)^{\frac{p}{2}} d x\right]^{\frac{1}{p}}\right\} \\
& \geq C(p)\left\{\left(\varepsilon_{n}^{p}-\varepsilon_{2^{m+2}-1}^{p}\right) \lambda_{n}^{p}+\sum_{\nu=m}^{\infty}\left(\varepsilon_{2^{\nu+2}-1}^{p}-\varepsilon_{2^{\nu+3}-1}^{p}\right) \lambda_{2^{\nu}}^{p}\right\}^{\frac{1}{p}} \\
& \geq C(p)\left\{\left(\varepsilon_{n}^{p}-\varepsilon_{2^{m+1}-1}^{p}\right) \lambda_{n}^{p}+\sum_{\nu=m+1}^{\infty}\left(\varepsilon_{2^{\nu}-1}^{p}-\varepsilon_{2^{\nu+1}-1}^{p}\right) \lambda_{2^{\nu}}^{p}\right\}^{\frac{1}{p}} \\
& \geq C(p)\left\{\varepsilon_{n}^{p} \lambda_{n}^{p}+\sum_{\nu=n+1}^{\infty}\left(\lambda_{\nu+1}^{p}-\lambda_{\nu}^{p}\right) \varepsilon_{\nu}^{p}\right\}^{\frac{1}{p}}
\end{aligned}
$$

This implies the necessity in (8) for $1<p<2$. The proof of the necessity part in (8) is complete.

STEP 6. Now we shall prove that $W_{p}^{\lambda, \beta} \subset E_{p}[\varepsilon]$ implies $\frac{1}{\lambda_{n}}=O\left[\varepsilon_{n}\right]$.

First, we note that the last condition is equivalent to the following one: $\forall \gamma=\left\{\gamma_{n}\right\} \in \Phi$ one has $\frac{\gamma_{n}}{\lambda_{n}}=O\left[\varepsilon_{n}\right]$. We shall obtain only nontrivial part which is : $\frac{\gamma_{n}}{\lambda_{n}}=O\left[\varepsilon_{n}\right]$ implies $\frac{1}{\lambda_{n}}=O\left[\varepsilon_{n}\right]$.

Let us assume $\frac{1}{\lambda_{n}}=O\left[\varepsilon_{n}\right]$ does not hold. Then there exists a sequence $\left\{C_{k} \uparrow \infty\right\}$ such that $\frac{1}{\lambda_{n_{k}} \varepsilon_{n_{k}}} \geq C_{k}$. Using $\frac{1}{\lambda_{n}}=O\left[\varepsilon_{n}\right]$ we have $\frac{C}{\gamma_{n_{k}}} \geq C_{k}$. Choosing $\gamma_{n_{k}}:=\frac{1}{\sqrt{C_{k}}} \rightarrow 0$, we write $C \geq \sqrt{C_{k}} \rightarrow \infty$. This contradiction gives

$$
\frac{1}{\lambda_{n}}=O\left[\varepsilon_{n}\right] \quad \Leftrightarrow \quad \forall \gamma=\left\{\gamma_{n}\right\} \in \Phi \quad \frac{\gamma_{n}}{\lambda_{n}}=O\left[\varepsilon_{n}\right] .
$$


Let us assume $\frac{\gamma_{n}}{\lambda_{n}}=O\left[\varepsilon_{n}\right]$ does not hold for all $\gamma \in \Phi$, but $W_{p}^{\lambda, \beta} \subset E_{p}[\varepsilon]$. Then there exist $\gamma=\left\{\gamma_{n}\right\} \in \Phi$ and $\left\{C_{n} \uparrow \infty\right\}$ such that $\frac{\gamma_{m_{n}}}{\lambda_{m_{n}}} \geq C_{n} \varepsilon_{m_{n}}$. Further, we choose a subsequence $\left\{m_{n_{k}}\right\}$ such that $\frac{m_{n_{k+1}}}{m_{n_{k}}} \geq 2$ and $\gamma_{m_{n_{k}}} \leq 2^{-k}$. Consider the series

$$
\sum_{k=0}^{\infty} \frac{\gamma_{m_{n_{k}}}}{\lambda_{m_{n_{k}}}} \cos \left(m_{n_{k}}+1\right) x
$$

Since

$$
\sum_{k=0}^{\infty} \frac{\gamma_{m_{n_{k}}}}{\lambda_{m_{n_{k}}}} \leq \frac{1}{\lambda_{m_{n_{0}}}} \sum_{k=0}^{\infty} \frac{1}{2^{k}}<\infty,
$$

there exists a function $f_{6} \in L_{p}$ with Fourier series (30). Because $\sum_{k=0}^{\infty} \gamma_{m_{n_{k}}} \leq \sum_{k=0}^{\infty} \frac{1}{2^{k}}<$ $\infty$, we have $f_{6}^{(\lambda, \beta)} \in L_{p}$, i.e. $f_{6} \in W_{p}^{\lambda, \beta}$. By (20) and by Lemma 1 ,

$$
\begin{aligned}
E_{m_{n_{k}}}\left(f_{6}\right)_{p} \geq C(p)\left\|f_{6}-S_{m_{n_{k}}}\left(f_{6}\right)\right\|_{p} \geq C(p)\left(\sum_{s=k}^{\infty} \frac{\gamma_{m_{n_{s}}}^{2}}{\lambda_{m_{n_{s}}}^{2}}\right)^{\frac{1}{2}} & \geq C(p) \frac{\gamma_{m_{n_{k}}}}{\lambda_{m_{n_{k}}}} \\
& \geq C(p) C_{n_{k}} \varepsilon_{m_{n_{k}}}
\end{aligned}
$$

i.e. $f_{6} \notin E_{p}[\varepsilon]$. This contradiction implies that the condition $\frac{1}{\lambda_{n}}=O\left[\varepsilon_{n}\right]$ is necessary for $W_{p}^{\lambda, \beta} \subset E_{p}[\varepsilon]$. The proof of the necessity part in (9) is complete.

STEP 7. Let us prove that $W_{p}^{\lambda, \beta} E[\omega] \subset E_{p}[\varepsilon]$ implies $\frac{\omega_{n}}{\lambda_{n}}=O\left[\varepsilon_{n}\right]$. If the last condition does not hold, then there exists $\left\{C_{n} \uparrow \infty\right\}$ such that $\frac{\omega_{m_{n}}}{\lambda_{m_{n}}} \geq C_{n} \varepsilon_{m_{n}}$. We choose a subsequence $\left\{m_{n_{k}}\right\}$ such that $\frac{m_{n_{k+1}}}{m_{n_{k}}} \geq 2$ and $\omega_{m_{n_{k}}} \geq \frac{1}{2} \omega_{m_{n_{k}}}+\omega_{m_{n_{k+1}}}$. Since

$$
\sum_{k=0}^{\infty} \frac{\omega_{m_{n_{k}}}^{2}-\omega_{m_{n_{k+1}}}^{2}}{\lambda_{m_{n_{k}}}^{2}} \leq \frac{\omega_{m_{n_{0}}}^{2}}{\lambda_{m_{n_{0}}}^{2}},
$$

by Lemma 1, the series

$$
\sum_{k=0}^{\infty} \frac{\left(\omega_{m_{n_{k}}}^{2}-\omega_{m_{n_{k+1}}}^{2}\right)^{\frac{1}{2}}}{\lambda_{m_{n_{k}}}} \cos \left(m_{n_{k}}+1\right) x
$$

is the Fourier series of a function $f_{7} \in L_{p}$. We have also $f_{7}^{(\lambda, \beta)} \in L_{p}, E_{n}\left(f_{7}^{(\lambda, \beta)}\right)_{p} \leq C \omega_{n}$.

On the other hand,

$$
\begin{aligned}
& E_{m_{n_{k}}}\left(f_{7}\right)_{p} \geq C(p)\left\|f_{7}-S_{m_{n_{k}}}\left(f_{7}\right)\right\|_{p} \geq C(p)\left(\sum_{s=k}^{\infty} \frac{\omega_{m_{n_{s}}}^{2}-\omega_{m_{n_{s+1}}}^{2}}{\lambda_{m_{n_{s}}}^{2}}\right)^{\frac{1}{2}} \\
= & C(p)\left(\sum_{s=k}^{\infty} \frac{\left(\omega_{m_{n_{s}}}-\omega_{m_{n_{s+1}}}\right)\left(\omega_{m_{n_{s}}}+\omega_{m_{n_{s+1}}}\right)}{\lambda_{m_{n_{s}}}^{2}}\right)^{\frac{1}{2}} \geq C(p) \frac{\omega_{m_{n_{k}}}}{\lambda_{m_{n_{k}}}} \geq C(p) C_{n_{k}} \varepsilon_{m_{n_{k}}},
\end{aligned}
$$

i.e. $f_{7} \notin E_{p}[\varepsilon]$. Therefore, $\frac{\omega_{n}}{\lambda_{n}}=O\left[\varepsilon_{n}\right]$. The proof of the necessity part in (10) is complete. 
6. Proof of Theorem 2. We divide the proof of Theorem 2 into two parts.

\subsection{Proof of sufficiency}

STEP 1. Let us show that if the series in (11) converges and $f \in E_{p}[\varepsilon]$, then $f \in W_{p}^{\lambda, \beta}$. We consider the series

$$
\begin{aligned}
& \cos \frac{\pi \beta}{2} V_{1}(\lambda, f)-\sin \frac{\pi \beta}{2} \widetilde{V_{1}}(\lambda, f) \\
+ & \sum_{n=1}^{\infty}\left\{\cos \frac{\pi \beta}{2}\left(V_{2^{n}}(\lambda, f)-V_{2^{n-1}}(\lambda, f)\right)-\sin \frac{\pi \beta}{2}\left(\widetilde{V_{2^{n}}}(\lambda, f)-\widetilde{V_{2^{n-1}}}(\lambda, f)\right)\right\},
\end{aligned}
$$

where $V_{1}(\lambda, f):=\lambda_{1} A_{1}(f, x)$,

$$
V_{n}(\lambda, f):=\sigma\left(\lambda, V_{n}(f)\right)=\sum_{m=1}^{n} \lambda_{m} A_{m}(f, x)+\sum_{m=n+1}^{2 n-1} \lambda_{m}\left(1-\frac{m-n}{n}\right) A_{m}(f, x)(n \geq 2) .
$$

Let $M>N>0$. From the inequality $\left\|f-V_{n}(f)\right\|_{p} \leq C E_{n}(f)_{p}$ and Lemma 7, using the properties of $\left\{\lambda_{n}\right\}$, we get

$$
\begin{aligned}
& \left.A:=\| \sum_{n=N}^{M}\left[\cos \frac{\pi \beta}{2}\left(V_{2^{n+1}}(\lambda, f)-V_{2^{n}}(\lambda, f)\right)-\sin \frac{\pi \beta}{2} \widetilde{\left(V_{2^{n+1}}\right.}(\lambda, f)-\widetilde{V_{2^{n}}}(\lambda, f)\right)\right] \|_{p} \\
& \leq \sum_{n=N}^{M}\left[\left|\cos \frac{\pi \beta}{2}\right|\left\|V_{2^{n+1}}(f)-V_{2^{n}}(f)\right\|_{p}\right. \\
& \times\left(\sum_{m=2^{n-1}-1}^{2^{n+2}+3}\left|\triangle^{2} \lambda_{m+2}\right|(m+1)+\left(2^{n+2}-1\right)\left|\triangle \lambda_{2^{n+2}-1}\right|\right) \\
& \left.+\left|\sin \frac{\pi \beta}{2}\right|\left\|\widetilde{V_{2^{n+1}}}(f)-\widetilde{V_{2^{n}}}(f)\right\|_{p}\left(\sum_{m=2^{n-1}-1}^{2^{n+2}+3}\left|\triangle^{2} \lambda_{m+2}\right|(m+1)+\left(2^{n+2}-1\right)\left|\triangle \lambda_{2^{n+2}-1}\right|\right)\right] \\
& +\left|\cos \frac{\pi \beta}{2}\right|\left\|\sum_{n=N}^{M} \lambda_{2^{n+1}-1}\left(V_{2^{n+1}}-V_{2^{n}}\right)(f)||_{p}+\left|\sin \frac{\pi \beta}{2}\right|\right\| \sum_{n=N}^{M} \lambda_{2^{n+1}-1}\left(\widetilde{V_{2^{n+1}}}-\widetilde{V_{2^{n}}}\right)(f) \|_{p} \\
& \leq C\left\{\lambda_{2^{N-1}}\left(\left|\cos \frac{\pi \beta}{2}\right| E_{2^{N-1}}(f)_{p}+\left|\sin \frac{\pi \beta}{2}\right| E_{2^{N-1}}(\tilde{f})_{p}\right)\right. \\
& \left.+\sum_{n=2^{N-1}}^{\infty}\left(\lambda_{n+1}-\lambda_{n}\right)\left(\left|\cos \frac{\pi \beta}{2}\right|_{n}(f)_{p}+\left|\sin \frac{\pi \beta}{2}\right| E_{n}(\tilde{f})_{p}\right)\right\} .
\end{aligned}
$$

Further, we apply Lemma 3 . Then the convergence of series in (11) and $f \in E_{p}[\varepsilon]$ imply that there exists $\varphi \in L_{p}$ such that the series (32) converges to $\varphi$ in $L_{p}$.

Let us show that $\sigma(\varphi)=\sigma\left(f^{(\lambda, \beta)}\right)$. If $F_{n}$ is the $n$-th partial sum of (32), then, say for cosine coefficients, $a_{n}(\varphi)=a_{n}\left(\varphi-F_{N+n}\right)+a_{n}\left(F_{N+n}\right)=a_{n}\left(\varphi-F_{N+n}\right)+a_{n}\left(f^{(\lambda, \beta)}\right)$, and

$$
a_{n}\left(\varphi-F_{N+n}\right)=\frac{1}{\pi} \int_{-\pi}^{\pi}\left(\varphi-F_{N+n}\right)(x) \cos n x d x \leq C(p)\left\|\varphi-F_{N+n}\right\|_{p} \rightarrow 0(N \rightarrow \infty) .
$$

The proof of the sufficiency part in (11) is complete.

STEP 2. Let us prove the sufficiency part in (12). Let $\sin \frac{\pi \beta}{2}=0$. If $n=0,1$, then the proof comes from (11). If $2^{m-1}+1 \leq n<2^{m}, m \in \mathbf{N}$, we consider the best approximant 
$T_{n}^{*}(x)=T_{n}^{*}(f, x)$, i.e. $E_{n}(f)_{p}=\left\|f(\cdot)-T_{n}^{*}(f, \cdot)\right\|_{p}$.

$$
\begin{aligned}
E_{n}\left(f^{(\lambda, \beta)}\right)_{p} & \leq\left\|f^{(\lambda, \beta)}-T_{n}^{*(\lambda, \beta)}+V_{2^{m+2}}\left(f^{(\lambda, \beta)}\right)-V_{2^{m+2}}\left(f^{(\lambda, \beta)}\right)\right\|_{p} \\
& \leq\left\|T_{n}^{*(\lambda, \beta)}-V_{2^{m+2}}\left(f^{(\lambda, \beta)}\right)\right\|_{p}+\left\|f^{(\lambda, \beta)}-V_{2^{m+2}}\left(f^{(\lambda, \beta)}\right)\right\|_{p} .
\end{aligned}
$$

By Lemma 7, we obtain

$$
\left\|T_{n}^{*(\lambda, \beta)}-V_{2^{m+2}}\left(f^{(\lambda, \beta)}\right)\right\|_{p} \leq C(\lambda) \lambda_{n}\left\|T_{n}^{*}-V_{2^{m+2}}(f)\right\|_{p} \leq C(\lambda) \lambda_{n} E_{n}(f)_{p} .
$$

Further, applying two times Abel's transformation and Lemma 7 we write for $M>N$

$$
\begin{aligned}
& A=\left\|\sum_{n=N}^{M}\left(V_{2^{n+1}}\left(f^{(\lambda, \beta)}\right)-V_{2^{n}}\left(f^{(\lambda, \beta)}\right)\right)\right\|_{p} \\
& \leq \sum_{n=N}^{M}\left\|V_{2^{n+1}}(f)-V_{2^{n}}(f)\right\|_{p}\left(\sum_{m=2^{n}+1}^{2^{n+2}-3}\left|\triangle^{2} \lambda_{m+2}\right|(m+1)+\left(2^{n+2}-1\right)\left|\lambda_{2^{n+2}-1}-\lambda_{2^{n+2}}\right|\right) \\
& +\left\|\sum_{n=N}^{M} \lambda_{2^{n+2}-1}\left(V_{2^{n+1}}-V_{2^{n}}\right)(f)\right\|_{p} \\
& \leq C(\lambda)\left\{\lambda_{2^{N+1}} E_{2^{N}}(f)_{p}+\sum_{n=N}^{\infty} E_{2^{n}}(f)_{p}\left(\lambda_{2^{n+2}-1}-\lambda_{2^{n+1}-1}\right)\right\} .
\end{aligned}
$$

Then

$$
\left\|f^{(\lambda, \beta)}-V_{2^{m+2}}\left(f^{(\lambda, \beta)}\right)\right\|_{p} \leq C(\lambda)\left\{\lambda_{n} E_{n}(f)_{p}+\sum_{\nu=n+1}^{\infty}\left(\lambda_{\nu+1}-\lambda_{\nu}\right) E_{\nu}(f)_{p}\right\} .
$$

Let $\sin \frac{\pi \beta}{2} \neq 0$. Then the convergence of series in (11) implies that there exist $\tilde{f} \in L_{p}$, $f^{(\lambda, 0)} \in L_{p}$ and $\widetilde{f}^{(\lambda, 0)} \in L_{p}$. Then

$$
\begin{aligned}
E_{n}\left(f^{(\lambda, \beta)}\right)_{p} & \leq E_{n}\left(\cos \frac{\pi \beta}{2} f^{(\lambda, 0)}-\sin \frac{\pi \beta}{2} \widetilde{f}^{(\lambda, 0)}\right)_{p} \\
& \leq\left|\cos \frac{\pi \beta}{2}\right| E_{n}\left(f^{(\lambda, 0)}\right)_{p}+\left|\sin \frac{\pi \beta}{2}\right| E_{n}\left(\widetilde{f}^{(\lambda, 0)}\right)_{p} .
\end{aligned}
$$

Applying (33), we get

$$
E_{n}\left(f^{(\lambda, 0)}\right)_{p} \leq C(\lambda)\left\{\lambda_{n} E_{n}(f)_{p}+\sum_{\nu=n+1}^{\infty}\left(\lambda_{\nu+1}-\lambda_{\nu}\right) E_{\nu}(f)_{p}\right\} .
$$

By Lemma 3, (33) implies

$$
\begin{aligned}
E_{n}\left(\widetilde{f}^{(\lambda, 0)}\right)_{p} & \leq C(\lambda)\left\{\lambda_{n} E_{n}(\widetilde{f})_{p}+\sum_{\nu=n+1}^{\infty}\left(\lambda_{\nu+1}-\lambda_{\nu}\right) E_{\nu}(\widetilde{f})_{p}\right\} \\
& \leq C(\lambda)\left\{\lambda_{n} E_{n}(f)_{p}+\sum_{\nu=n+1}^{\infty} \frac{\lambda_{\nu}}{\nu} E_{\nu}(f)_{p}\right\} .
\end{aligned}
$$


Therefore, for all $\beta \in \mathbf{R}$ we have

$$
\begin{array}{r}
E_{n}\left(f^{(\lambda, \beta)}\right)_{p} \leq C(\lambda, \beta)\left\{\lambda_{n} E_{n}(f)_{p}+\left|\cos \frac{\pi \beta}{2}\right| \sum_{\nu=n+1}^{\infty}\left(\lambda_{\nu+1}-\lambda_{\nu}\right) E_{\nu}(f)_{p}\right. \\
\left.+\left|\sin \frac{\pi \beta}{2}\right| \sum_{\nu=n+1}^{\infty} \frac{\lambda_{\nu}}{\nu} E_{\nu}(f)_{p}\right\} .
\end{array}
$$

If $f \in E_{p}[\varepsilon]$, from (12) we obtain $E_{n}\left(f^{(\lambda, \beta)}\right)_{p}=O\left(\omega_{n}\right)$, i.e. $f \in W_{p}^{\lambda, \beta} E[\omega]$. The proof of the sufficiency part in (12) is complete.

STEP 3. Let us show that if $\frac{1}{\lambda_{n}}=O\left(\varepsilon_{n}\right)$ and $f \in W_{p}^{\lambda, \beta}$, then $f \in E_{p}[\varepsilon]$. Also we shall verify that if $\frac{\omega}{\lambda_{n}}=O\left(\varepsilon_{n}\right)$ and $f \in W_{p}^{\lambda, \beta} E[\omega]$, then $f \in E_{p}[\varepsilon]$. Set $\frac{1}{\lambda}:=\left\{\frac{1}{\lambda_{1}}, \frac{1}{\lambda_{2}}, \frac{1}{\lambda_{3}}, \ldots\right\}$.

Let $\sin \frac{\pi \beta}{2}=0$. Applying two times Abel's transformation and Lemma 7 we have for $M>N$

$$
\begin{aligned}
& A:=\left\|\sum_{n=N}^{M}\left[V_{2^{n+1}}(f)-V_{2^{n}}(f)\right]\right\|_{p}=\left\|\sum_{n=N}^{M}\left[\left(V_{2^{n+1}}\left(f^{(\lambda, \beta)}\right)\right)^{\left(\frac{1}{\lambda}, 0\right)}-\left(V_{2^{n}}\left(f^{(\lambda, \beta)}\right)\right)^{\left(\frac{1}{\lambda}, 0\right)}\right]\right\|_{p} \\
& \leq \sum_{n=N}^{M}\left\|V_{2^{n+1}}\left(f^{(\lambda, \beta)}\right)-V_{2^{n}}\left(f^{(\lambda, \beta)}\right)\right\|_{p} \\
& \quad \times\left(\sum_{m=2^{n}+1}^{2^{n+2}-3}\left|\triangle^{2} \lambda_{m+2}^{-1}\right|(m+1)+\left(2^{n+2}-1\right)\left|\lambda_{2^{n+2}-2}^{-1}-\lambda_{2^{n+2}-1}^{-1}\right|\right) \\
& +\left\|\sum_{n=N}^{M} \lambda_{2^{n+2}-1}^{-1}\left[V_{2^{n+1}}\left(f^{(\lambda, \beta)}\right)-V_{2^{n}}\left(f^{(\lambda, \beta)}\right)\right]\right\|_{p} \leq \frac{C(\lambda)}{\lambda_{2^{N}}}\left\|f^{(\lambda, \beta)}\right\|_{p} .
\end{aligned}
$$

Then, from (34), one has

$$
E_{n}(f)_{p} \leq \frac{C(\lambda)}{\lambda_{2^{N}}}\left\|f^{(\lambda, \beta)}\right\|_{p}
$$

Let $T_{n}^{*}(x)$ be the best approximant for $f^{(\lambda, \beta)}$, i.e. $E_{n}\left(f^{(\lambda, \beta)}\right)_{p}=\left\|f^{(\lambda, \beta)}(\cdot)-T_{n}^{*}(\cdot)\right\|_{p}$. Using (35) for $f-T_{n}^{*\left(\frac{1}{\lambda},-\beta\right)}$, we get

$$
E_{n}(f)_{p}=E_{n}\left(f^{(\lambda, \beta)}-T_{n}^{*\left(\frac{1}{\lambda},-\beta\right)}\right)_{p} \leq \frac{C(\lambda)}{\lambda_{n}}\left\|f^{(\lambda, \beta)}(\cdot)-T_{n}^{*}(\cdot)\right\|_{p}=\frac{C(\lambda)}{\lambda_{n}} E_{n}\left(f^{(\lambda, \beta)}\right)_{p} .
$$

Let $\sin \frac{\pi \beta}{2} \neq 0$. Then

$$
\begin{aligned}
& A=\| \sum_{n=N}^{M}\left[\cos \frac{\pi \beta}{2}\left(\left(V_{2^{n+1}}\left(f^{(\lambda, \beta)}\right)\right)^{\left(\frac{1}{\lambda}, 0\right)}-\left(V_{2^{n}}\left(f^{(\lambda, \beta)}\right)\right)^{\left(\frac{1}{\lambda}, 0\right)}\right)\right. \\
& \left.+\sin \frac{\pi \beta}{2}\left(\left(\widetilde{V}_{2^{n+1}}\left(f^{(\lambda, \beta)}\right)\right)^{\left(\frac{1}{\lambda}, 0\right)}-\left(\widetilde{V}_{2^{n}}\left(f^{(\lambda, \beta)}\right)\right)^{\left(\frac{1}{\lambda}, 0\right)}\right)\right] \|_{p} \\
& \leq\left\|\cos \frac{\pi \beta}{2} \sum_{n=N}^{M}\left[\left(V_{2^{n+1}}\left(f^{(\lambda, \beta)}\right)\right)^{\left(\frac{1}{\lambda}, 0\right)}-\left(V_{2^{n}}\left(f^{(\lambda, \beta)}\right)\right)^{\left(\frac{1}{\lambda}, 0\right)}\right]\right\|_{p} \\
& +\left\|\sin \frac{\pi \beta}{2} \sum_{n=N}^{M}\left[\left(\widetilde{V}_{2^{n+1}}\left(f^{(\lambda, \beta)}\right)\right)^{\left(\frac{1}{\lambda}, 0\right)}-\left(\widetilde{V}_{2^{n}}\left(f^{(\lambda, \beta)}\right)\right)^{\left(\frac{1}{\lambda}, 0\right)}\right]\right\|_{p} .
\end{aligned}
$$


To estimate the first item we apply (34) and to estimate the second one we use Lemmas 7,8 , as well as the condition $\sum_{\nu=n+1}^{\infty} \frac{1}{\nu \lambda_{\nu}} \leq \frac{C}{\lambda_{n}}$. We get

$$
\left\|\sum_{n=N}^{M} V_{2^{n+1}}\left(f^{(\lambda, \beta)}\right)-V_{2^{n}}\left(f^{(\lambda, \beta)}\right)\right\|_{p} \leq \frac{C(\lambda, \beta)}{\lambda_{2^{N}}}\left\|f^{(\lambda, \beta)}\right\|_{p} .
$$

Repeating the argument we used in proving (36) we arrive at the inequality

$$
E_{n}(f)_{p} \leq \frac{C(\lambda, \beta)}{\lambda_{n}} E_{n}\left(f^{(\lambda, \beta)}\right)_{p}
$$

From this it is clear that $\frac{1}{\lambda_{n}}=O\left(\varepsilon_{n}\right)$ and $\frac{\omega_{n}}{\lambda_{n}}=O\left(\varepsilon_{n}\right)$ are necessary conditions for $W_{p}^{\lambda, \beta} \subset E_{p}[\varepsilon]$ and $W_{p}^{\lambda, \beta} E(\omega) \subset E_{p}[\varepsilon]$, respectively. The proofs of the sufficiency parts in (13) and (14) are complete.

\subsection{Proof of necessity}

STEP 4. Let us show that if $E_{p}[\varepsilon] \subset W_{p}^{\lambda, \beta}$, then the series in (11) converges. Suppose that it diverges.

SteP 4(a): $p=\infty$. We start with the case $\sin \frac{\pi \beta}{2}=0$. Then we define the series

$$
\sum_{\nu=1}^{\infty}\left(\varepsilon_{\nu-1}-\varepsilon_{\nu}\right)(\cos \nu x+\sin \nu x)
$$

which converges to the function $f_{8} \in L_{p}$. It can be easily found: $E_{n}\left(f_{8}\right)_{p} \leq \varepsilon_{n}$, i.e. $f_{8} \in E_{p}[\varepsilon] \subset W_{p}^{\lambda, \beta}$. On the other hand,

$$
\begin{aligned}
\left\|f_{8}^{(\lambda, \beta)}\right\|_{p} & \geq \sum_{\nu=1}^{\infty} \lambda_{\nu}\left(\varepsilon_{\nu-1}-\varepsilon_{\nu}\right)=\lambda_{1}\left(\varepsilon_{0}-\varepsilon_{1}\right)+\sum_{\nu=2}^{\infty}\left(\varepsilon_{\nu-1}-\varepsilon_{\nu}\right)\left[\sum_{m=2}^{\nu}\left(\lambda_{m}-\lambda_{m-1}\right)+\lambda_{1}\right] \\
& =\sum_{m=2}^{\infty}\left(\lambda_{m}-\lambda_{m-1}\right) \varepsilon_{m-1}+\lambda_{1} \varepsilon_{0}=\sum_{n=1}^{\infty}\left(\lambda_{n+1}-\lambda_{n}\right) \varepsilon_{n}+\lambda_{1} \varepsilon_{0}=\infty
\end{aligned}
$$

This contradiction implies the convergence of the series in (11).

Let $\sin \frac{\pi \beta}{2} \neq 0$. Since

$$
\sum_{n=1}^{\infty}\left(\lambda_{n+1}-\lambda_{n}\right) \varepsilon_{n} \leq C \sum_{\nu=0}^{\infty} \lambda_{2^{\nu}} \varepsilon_{2^{\nu}} \leq C\left(\sum_{\nu=1}^{\infty} \varepsilon_{2^{\nu}} \sum_{m=2^{\nu-1}}^{2^{\nu}-1} \frac{\lambda_{m}}{m}+\lambda_{1} \varepsilon_{1}\right) \leq C \sum_{n=1}^{\infty} \frac{\lambda_{n}}{n} \varepsilon_{n},
$$

we have

$$
\left|\cos \frac{\beta \pi}{2}\right| \sum_{n=1}^{\infty}\left(\lambda_{n+1}-\lambda_{n}\right) \varepsilon_{n}+\left|\sin \frac{\beta \pi}{2}\right| \sum_{n=1}^{\infty} \lambda_{n} \frac{\varepsilon_{n}}{n} \asymp \sum_{n=1}^{\infty} \lambda_{n} \frac{\varepsilon_{n}}{n} .
$$

We consider the series $\sum_{n=1}^{\infty} \frac{\varepsilon_{n-1}}{n} \sin n x$. Then, by Lemma 4 , the sum of this series, say, $f_{9}(x)$ is in $L_{\infty}$. From [Ba1], $E_{n}\left(f_{9}\right)_{p} \leq C \varepsilon_{n}$, i.e. $f_{9} \in E_{p}[\varepsilon] \subset W_{p}^{\lambda, \beta}$. We also have

$$
\left\|f_{9}^{(\lambda, \beta)}\right\|_{p} \geq C \sum_{n=1}^{\infty} \frac{\varepsilon_{n-1}}{n} \lambda_{n} \geq C \sum_{n=1}^{\infty} \frac{\varepsilon_{n}}{n} \lambda_{n}=\infty .
$$

Thus, the series in (11) converges. 
STEP $4(\mathrm{~b}): p=1$. First let $\sin \frac{\pi \beta}{2}=0$. We define the series

$$
\sum_{\nu=1}^{\infty}\left(\varepsilon_{\nu-1}-\varepsilon_{\nu}\right) \tau_{\nu}(x)
$$

where

$$
\tau_{\nu+1}(x)=\sum_{j=1}^{\nu+1} \alpha_{j}^{\nu} \sin j x \quad \text { and } \quad \alpha_{j}^{\nu}=\left\{\begin{array}{ll}
\frac{j}{\nu+2}, & 1 \leq j \leq \frac{\nu+2}{2} \\
1-\frac{j}{\nu+2}, & \frac{\nu+2}{2} \leq j \leq \nu+1
\end{array} .\right.
$$

The series $(38)$ converges to a $f_{10} \in L_{p}$ and $E_{n}\left(f_{10}\right)_{p} \leq C \varepsilon_{n}$ (see [Ge]). Then $f_{10} \in$ $E_{p}[\varepsilon] \subset W_{p}^{\lambda, \beta}$. One can rewrite $(38)$ in the following way:

$$
\sum_{\nu=1}^{\infty} b_{\nu} \sin \nu x, \quad \text { where } \quad b_{\nu}=\sum_{j=\nu}^{2 \nu-2}\left(1-\frac{\nu}{j+1}\right)\left(\varepsilon_{\nu-1}-\varepsilon_{\nu}\right)+\sum_{j=2 \nu-1}^{\infty} \frac{\nu}{j+1}\left(\varepsilon_{\nu-1}-\varepsilon_{\nu}\right) .
$$

By Lemma 5, we get

$$
\left\|f_{10}^{(\lambda, \beta)}\right\|_{1} \geq C \sum_{\nu=1}^{\infty} \lambda_{\nu} \frac{b_{\nu}}{\nu} \geq C\left(\sum_{\nu=2}^{\infty}\left(\lambda_{\nu+1}-\lambda_{\nu}\right) \varepsilon_{\nu}+\lambda_{3} \varepsilon_{3}\right)=\infty .
$$

This contradicts the divergence of the series in (11). Thus, the series in (11) converges.

Let $\sin \frac{\pi \beta}{2} \neq 0$. As we saw in (37), the divergence of the series in (11) in this case is equivalent to the divergence of $\sum_{n=1}^{\infty} \frac{\lambda_{n}}{n} \varepsilon_{n}$.

We consider the series

$$
\sum_{\nu=1}^{\infty}\left(\varepsilon_{\nu-1}-\varepsilon_{\nu}\right) K_{\nu}(x)
$$

This series is convergent in $L_{1}$ (see [Ge]) to a function $f_{11}(x)$, and $E_{n}\left(f_{11}\right)_{p}=O\left(\varepsilon_{n}\right)$. Therefore, $f_{10} \in E_{p}[\varepsilon] \subset W_{p}^{\lambda, \beta}$.

One can rewrite (39) in the following way:

$$
\sum_{\nu=1}^{\infty} a_{\nu} \cos \nu x, \quad \text { where } \quad a_{\nu}=\varepsilon_{\nu-1}-\nu \sum_{j=\nu}^{\infty} \frac{\varepsilon_{j-1}-\varepsilon_{j}}{j+1} .
$$

We note that $\left\{a_{\nu}\right\}$ is a monotonic null sequence. Indeed,

$$
a_{\nu}-a_{\nu+1}=\sum_{j=\nu}^{\infty} \frac{\varepsilon_{j-1}-\varepsilon_{j}}{j+1} \geq 0 .
$$

By Lemma 5, using monotonicity of $\left\{a_{\nu}\right\}$ and the conditions on $\left\{\lambda_{\nu}\right\}$, we have

$$
\begin{aligned}
\left\|f_{11}^{(\lambda, \beta)}\right\|_{1} & \geq C \sum_{\nu=1}^{\infty} \frac{\lambda_{\nu}}{\nu} a_{\nu} \geq C \sum_{\nu=1}^{\infty} \lambda_{2^{\nu+1}} a_{2^{\nu}}=C \sum_{\nu=1}^{\infty} a_{2^{\nu}}\left[\sum_{n=1}^{\nu}\left(\lambda_{2^{n+1}}-\lambda_{2^{n}}\right)+\lambda_{2}\right] \\
& \geq C\left(\lambda_{1} a_{1}+\sum_{n=1}^{\infty}\left(\lambda_{2^{n+1}}-\lambda_{2^{n}}\right) a_{2^{n}}\right) \geq C\left(\lambda_{1} a_{1}+\sum_{n=2}^{\infty} a_{2^{n}} \sum_{\nu=2^{n}}^{2^{n+1}-1}\left(\lambda_{\nu+1}-\lambda_{\nu}\right)\right) \\
& \geq C\left(\lambda_{1} a_{1}+\sum_{n=1}^{\infty}\left(\lambda_{n+1}-\lambda_{n}\right) a_{n}\right) .
\end{aligned}
$$


On the other hand, by (40), we have

$$
\begin{aligned}
\left\|f_{11}^{(\lambda, \beta)}\right\|_{1} & \geq C \sum_{\nu=1}^{\infty} \frac{\lambda_{\nu}}{\nu} a_{\nu}=C\left(\sum_{\nu=1}^{\infty} \frac{\lambda_{\nu}}{\nu} \varepsilon_{\nu-1}-\sum_{\nu=1}^{\infty} \lambda_{\nu} \sum_{j=\nu}^{\infty} \frac{\varepsilon_{j-1}-\varepsilon_{j}}{j+1}\right) \\
& \geq C\left(\sum_{\nu=1}^{\infty} \frac{\lambda_{\nu}}{\nu} \varepsilon_{\nu-1}-\sum_{\nu=1}^{\infty}\left(a_{\nu}-a_{\nu+1}\right) \lambda_{\nu+1}\right) \\
& =C\left(\sum_{\nu=1}^{\infty} \frac{\lambda_{\nu}}{\nu} \varepsilon_{\nu-1}-\sum_{\nu=1}^{\infty}\left(a_{\nu}-a_{\nu+1}\right)\left[\sum_{n=1}^{\nu}\left(\lambda_{n+1}-\lambda_{n}\right)+\lambda_{1}\right]\right) \\
& =C\left(\sum_{\nu=1}^{\infty} \frac{\lambda_{\nu}}{\nu} \varepsilon_{\nu-1}-\left[\lambda_{1} a_{1}+\sum_{n=1}^{\infty}\left(\lambda_{n+1}-\lambda_{n}\right) a_{n}\right]\right) .
\end{aligned}
$$

Combining this inequality and (41), we get

$$
\left\|f_{11}^{(\lambda, \beta)}\right\|_{1} \geq C \sum_{\nu=1}^{\infty} \lambda_{\nu} \nu^{-1} \varepsilon_{\nu-1}=\infty,
$$

and so, the series in (11) converges. The proof of the necessity part in (11) is complete.

STEP 5. Let us show the correctness of the necessity part in (12).

STEP 5(a): $p=\infty$. We consider the series

$$
\varepsilon_{n} \cos \left((n+1) x-\frac{\pi \beta}{2}\right)+\sum_{\nu=1}^{\infty}\left(\cos \frac{\pi \beta}{2}\left(\varepsilon_{\nu-1}-\varepsilon_{\nu}\right) \cos \nu x+\sin \frac{\pi \beta}{2} \frac{\varepsilon_{\nu-1}}{\nu} \sin \nu x\right)
$$

There exists a function $f_{12, n} \in L_{p}$ with the Fourier series (42). One can see $E_{m}\left(f_{12, n}\right)_{p} \leq$ $\varepsilon_{m}$, i.e. $f_{12, n} \in E_{p}[\varepsilon] \subset W_{p}^{\lambda, \beta} E[\omega]$. Therefore, $f_{12, n}^{(\lambda, \beta)} \in L_{p}$. Note that the series

$$
\sum_{\nu=1}^{\infty}\left(\cos \frac{\pi \beta}{2}\left(\varepsilon_{\nu-1}-\varepsilon_{\nu}\right) \cos \nu x+\sin \frac{\pi \beta}{2} \frac{\varepsilon_{\nu-1}}{\nu} \sin \nu x\right)
$$

is the Fourier series of a function $f_{13} \in L_{p}$ and $f_{13} \in E_{p}[\varepsilon] \subset W_{p}^{\lambda, \beta} E[\omega]$, i.e. $E_{m}\left(f_{13}\right)_{p}=$ $O\left(\omega_{m}\right)$.

Let us show that the positive constant $C_{1}$ in the inequality $E_{m}\left(f_{12, n}^{(\lambda, \beta)}\right)_{p} \leq C_{1} \omega_{m}$ $(m=0,1,2, \ldots)$ does not depend on $m$ and $n$. We have

$$
f_{12, n}(x)=f_{13}(x)+\varepsilon_{n} \cos \left((n+1) x-\frac{\pi \beta}{2}\right) .
$$

Let $m>n$. It is easy to see that

$$
f_{12, n}^{(\lambda, \beta)}(x)=f_{13}^{(\lambda, \beta)}(x)+\lambda_{n} \varepsilon_{n} \cos (n+1) x .
$$

Then, $E_{m}\left(f_{12, n}^{(\lambda, \beta)}\right)_{p} \leq E_{m}\left(f_{13}^{(\lambda, \beta)}\right)_{p} \leq C_{1}\left(f_{13}, \lambda, \beta\right) \omega_{m}$. We write for $0 \leq m \leq n$ :

$$
E_{m}\left(f_{12, n}^{(\lambda, \beta)}\right)_{p} \leq E_{m}\left(f_{13}^{(\lambda, \beta)}\right)_{p}+E_{m}\left(\lambda_{n} \varepsilon_{n} \cos (n+1) x\right)_{p} \leq C_{1}\left(f_{13}, \lambda, \beta\right) \omega_{m}+C_{2} \lambda_{n} \varepsilon_{n} .
$$

Hence, by Lemma $6, E_{m}\left(f_{13, n}^{(\lambda, \beta)}\right)_{p} \leq C \omega_{m}$ where $C$ does not depend on $n$ and $m$. 
Then

$$
\begin{array}{r}
C \omega_{n} \geq E_{n}\left(f_{12, n}^{(\lambda, \beta)}\right)_{p} \geq C\left[\lambda_{n} \varepsilon_{n}+\cos ^{2} \frac{\pi \beta}{2} \sum_{\nu=n+1}^{\infty}\left(\varepsilon_{\nu-1}-\varepsilon_{\nu}\right) \lambda_{\nu}+\sin ^{2} \frac{\pi \beta}{2} \sum_{\nu=n+1}^{\infty} \frac{\lambda_{\nu}}{\nu} \varepsilon_{\nu-1}\right] \\
\geq C\left[\lambda_{n} \varepsilon_{n}+\cos ^{2} \frac{\pi \beta}{2} \lambda_{n}\left(\sum_{\nu=n}^{2 n}\left(\varepsilon_{\nu}-\varepsilon_{\nu+1}\right)+\varepsilon_{2 n+1}\right)+\cos ^{2} \frac{\pi \beta}{2} \sum_{\nu=2 n+1}^{\infty} \lambda_{\nu}\left(\varepsilon_{\nu-1}-\varepsilon_{\nu}\right)\right. \\
\left.+\sin ^{2} \frac{\pi \beta}{2} \lambda_{n} \sum_{\nu=n}^{2 n} \frac{\varepsilon_{\nu}}{\nu}+\sin ^{2} \frac{\pi \beta}{2} \sum_{\nu=2 n+1}^{\infty} \lambda_{\nu} \frac{\varepsilon_{\nu-1}}{\nu}\right] \\
\geq C\left[\lambda_{n} \varepsilon_{n}+\left|\cos \frac{\pi \beta}{2}\right| \sum_{\nu=n+1}^{\infty}\left(\lambda_{\nu+1}-\lambda_{\nu}\right) \varepsilon_{\nu}+\left|\sin \frac{\pi \beta}{2}\right| \sum_{\nu=n+1}^{\infty} \lambda_{\nu} \frac{\varepsilon_{\nu}}{\nu}\right] .
\end{array}
$$

Thus, the relation in the right-hand side of (12) holds.

SteP 5(b): $p=1$. In this case we define the series

$$
\begin{aligned}
\varepsilon_{n} \quad & \sin \left((n+1) x-\frac{\pi \beta}{2}\right) \\
& +\sum_{\nu=1}^{\infty}\left(-\sin \frac{\pi \beta}{2}\left(\varepsilon_{\nu-1}-\varepsilon_{\nu}\right) K_{\nu}(x)+\cos \frac{\pi \beta}{2}\left(\varepsilon_{\nu-1}-\varepsilon_{\nu}\right) \tau_{\nu}(x)\right) .
\end{aligned}
$$

Then there exists a function $f_{14, n} \in L_{p}$ such that (43) is the Fourier series of $f_{14, n}$ (see $[\mathrm{Ge}])$. Also, $E_{m}\left(f_{14, n}\right)_{p}=O\left(\varepsilon_{m}\right)$, i.e. $f_{14, n} \in E_{p}[\varepsilon] \subset W_{p}^{\lambda, \beta} E[\omega]$. Note that the series

$$
\sum_{\nu=1}^{\infty}\left(-\sin \frac{\pi \beta}{2}\left(\varepsilon_{\nu-1}-\varepsilon_{\nu}\right) K_{\nu}(x)+\cos \frac{\pi \beta}{2}\left(\varepsilon_{\nu-1}-\varepsilon_{\nu}\right) \tau_{\nu}(x)\right)
$$

is the Fourier series of a function $f_{15} \in L_{p}$ and $f_{15} \in E_{p}[\varepsilon] \subset W_{p}^{\lambda, \beta} E[\omega]$, i.e. $E_{m}\left(f_{15}\right)_{p}=$ $O\left(\omega_{m}\right)$.

We shall prove that the positive constant $C_{2}$ in the inequality $E_{m}\left(f_{14, n}^{(\lambda, \beta)}\right)_{p} \leq C_{2} \omega_{m}$ $(m=0,1,2, \ldots)$ does not depend on $m$ and $n$. We note

$$
f_{14, n}^{(\lambda, \beta)}(x)=f_{15}^{(\lambda, \beta)}(x)+\lambda_{n} \varepsilon_{n} \sin (n+1) x .
$$

Let $m>n$. Then $E_{m}\left(f_{14, n}^{(\lambda, \beta)}\right)_{p}=E_{m}\left(f_{15}^{(\lambda, \beta)}\right)_{p} \leq C\left(f_{15}, \lambda, \beta\right) \omega_{m}$. For $0 \leq m \leq n$ we write

$$
E_{m}\left(f_{14, n}^{(\lambda, \beta)}\right)_{p} \leq E_{m}\left(f_{15}^{(\lambda, \beta)}\right)_{p}+E_{m}\left(\lambda_{n} \varepsilon_{n} \sin (n+1) x\right)_{p} \leq C\left(f_{15}, \lambda, \beta\right) \omega_{m}+C \lambda_{n} \varepsilon_{n} .
$$

Therefore, we have $E_{m}\left(f_{14, n}^{(\lambda, \beta)}\right)_{p} \leq C_{2} \omega_{m}$ from Lemma 6 .

We rewrite the series (43) in the following way :

$$
\varepsilon_{n} \sin \left((n+1) x-\frac{\pi \beta}{2}\right)+\sum_{\nu=1}^{\infty}\left(-\sin \frac{\pi \beta}{2} a_{\nu} \cos \nu x+\cos \frac{\pi \beta}{2} b_{\nu} \sin \nu x\right),
$$

where

$$
\begin{aligned}
a_{\nu} & =\varepsilon_{\nu-1}-\nu \sum_{j=\nu}^{\infty} \frac{\varepsilon_{j-1}-\varepsilon_{j}}{j+1}, \\
b_{\nu} & =\sum_{j=\nu}^{2 \nu-2}\left(1-\frac{\nu}{j+1}\right)\left(\varepsilon_{j-1}-\varepsilon_{j}\right)+\sum_{j=2 \nu-1}^{\infty} \frac{\nu}{j+1}\left(\varepsilon_{\nu-1}-\varepsilon_{\nu}\right) .
\end{aligned}
$$


We have

$$
\begin{aligned}
f_{14, n}^{(\lambda, \beta)}(x) & \sim \lambda_{n} \varepsilon_{n} \sin (n+1) x+\sum_{\nu=1}^{\infty} \lambda_{\nu}\left(-\sin \frac{\pi \beta}{2} \cos \frac{\pi \beta}{2} a_{\nu} \cos \nu x\right. \\
& \left.+\sin ^{2} \frac{\pi \beta}{2} a_{\nu} \sin \nu x+\cos ^{2} \frac{\pi \beta}{2} b_{\nu} \sin \nu x+\sin \frac{\pi \beta}{2} \cos \frac{\pi \beta}{2} b_{\nu} \cos \nu x\right) .
\end{aligned}
$$

We note that if $f(x) \sim c_{0}(f) / 2+\sum_{\nu=1}^{\infty}\left(c_{\nu}(f) \cos \nu x+d_{\nu}(f) \sin \nu x\right)$, then $E_{n}(f)_{p} \geq$ $C d_{n+1}(f)$. Then

$$
E_{n}\left(f_{14, n}^{(\lambda, \beta)}\right)_{p} \geq C\left(\lambda_{n} \varepsilon_{n}+\sin ^{2} \frac{\pi \beta}{2} \lambda_{n+1} a_{n+1}+\cos ^{2} \frac{\pi \beta}{2} \lambda_{n+1} b_{n+1}\right) \geq C \lambda_{n} \varepsilon_{n}
$$

Also $E_{n}\left(f_{14, n}^{(\lambda, \beta)}\right)_{p} \geq C \sin ^{2} \frac{\pi \beta}{2} \lambda_{n+1} a_{n+1}$ and, by Lemma 5 , we write for $2^{m-1} \leq n<2^{m}$

$$
\begin{aligned}
E_{n}\left(f_{14, n}^{(\lambda, \beta)}\right)_{p} & \geq C \sin ^{2} \frac{\pi \beta}{2} \sum_{\nu=n+1}^{\infty} \lambda_{\nu} \frac{a_{\nu}}{\nu} \geq C \sin ^{2} \frac{\pi \beta}{2}\left(\sum_{\nu=n+1}^{2^{m}-1} \lambda_{\nu} \frac{a_{\nu}}{\nu}+\sum_{k=m}^{\infty} \lambda_{2^{k+1}} a_{2^{k}}\right) \\
& \geq C \sin ^{2} \frac{\pi \beta}{2} \sum_{\nu=m}^{\infty}\left(\lambda_{2^{\nu+1}}-\lambda_{2^{\nu}}\right) \sum_{k=\nu}^{\infty} a_{2^{k}} \geq C \sin ^{2} \frac{\pi \beta}{2} \sum_{\nu=m}^{\infty}\left(\lambda_{2^{\nu+1}}-\lambda_{2^{\nu}}\right) a_{2^{\nu}} \\
& \geq C \sin ^{2} \frac{\pi \beta}{2} \sum_{\nu=2^{m}}^{\infty}\left(\lambda_{\nu+1}-\lambda_{\nu}\right) a_{\nu} .
\end{aligned}
$$

Hence,

$$
E_{n}\left(f_{14, n}^{(\lambda, \beta)}\right)_{p} \geq C \sin ^{2} \frac{\pi \beta}{2}\left(\lambda_{n+1} a_{n+1}+\sum_{\nu=n+1}^{\infty}\left(\lambda_{\nu+1}-\lambda_{\nu}\right) a_{\nu}\right) .
$$

On the other hand,

$$
\begin{aligned}
E_{n}\left(f_{14, n}^{(\lambda, \beta)}\right)_{p} & \geq C \sin ^{2} \frac{\pi \beta}{2} \sum_{\nu=n+1}^{\infty} \lambda_{\nu} \frac{a_{\nu}}{\nu} \\
& \geq C \sin ^{2} \frac{\pi \beta}{2}\left(\sum_{\nu=n+1}^{\infty} \frac{\lambda_{\nu}}{\nu} \varepsilon_{\nu-1}-\sum_{\nu=n+1}^{\infty}\left(a_{\nu}-a_{\nu+1}\right) \lambda_{\nu+1}\right) \\
& \geq C \sin ^{2} \frac{\pi \beta}{2}\left(\sum_{\nu=n+1}^{\infty} \frac{\lambda_{\nu}}{\nu} \varepsilon_{\nu-1}-C_{1}\left[\lambda_{n+1} a_{n+1}+\sum_{\nu=n+1}^{\infty}\left(\lambda_{\nu+1}-\lambda_{\nu}\right) a_{\nu}\right]\right) .
\end{aligned}
$$

Applying (45), we get

$$
E_{n}\left(f_{14, n}^{(\lambda, \beta)}\right)_{p} \geq C \sin ^{2} \frac{\pi \beta}{2} \sum_{\nu=n+1}^{\infty} \frac{\lambda_{\nu}}{\nu} \varepsilon_{\nu-1}
$$

Hence, using (44), (46), and Lemma 5, we have

$$
\begin{aligned}
E_{n}\left(f_{14, n}^{(\lambda, \beta)}\right)_{p} & \geq C\left(\lambda_{n} \varepsilon_{n}+\cos ^{2} \frac{\pi \beta}{2} \sum_{\nu=n+1}^{\infty} \lambda_{\nu} \frac{b_{\nu}}{\nu}+\sin ^{2} \frac{\pi \beta}{2} \sum_{\nu=n+1}^{\infty} \lambda_{\nu} \frac{a_{\nu}}{\nu}\right) \\
& \geq C\left(\lambda_{n} \varepsilon_{n}+\cos ^{2} \frac{\pi \beta}{2} \sum_{\nu=n+1}^{\infty} \varepsilon_{\nu}\left(\lambda_{\nu+1}-\lambda_{\nu}\right)+\sin ^{2} \frac{\pi \beta}{2} \sum_{\nu=n+1}^{\infty} \frac{\lambda_{\nu}}{\nu} \varepsilon_{\nu}\right) .
\end{aligned}
$$

This proves the necessity part in (12). 
STEP 6. Let us show that $W_{p}^{\lambda, \beta} \subset E_{p}[\varepsilon]$ implies $\frac{1}{\lambda_{n}}=O\left[\varepsilon_{n}\right]$. As we noticed above (see (29)) it is enough to show that $W_{p}^{\lambda, \beta} \subset E_{p}[\varepsilon]$ implies $\frac{\gamma_{n}}{\lambda_{n}}=O\left[\varepsilon_{n}\right]$ for all $\gamma=\left\{\gamma_{n}\right\} \in \Phi$.

We suppose that the last condition does not hold. Then there exist $\gamma=\left\{\gamma_{n}\right\} \in \Phi$ and $\left\{C_{n} \uparrow \infty\right\}$ such that $\frac{\gamma_{m_{n}}}{\lambda_{m_{n}}} \geq C_{n} \varepsilon_{m_{n}}$. We can choose a subsequence $\left\{m_{n_{k}}\right\}$ such that $\frac{m_{n_{k+1}}}{m_{n_{k}}} \geq 2$ and $\gamma_{m_{n_{k}}} \leq 2^{-k}$. Further, we consider the series (30), which is the Fourier series of $f_{6} \in L_{p}$ and $f_{6}^{(\lambda, \beta)} \in L_{p}$. On the other hand,

$$
E_{m_{n_{k}}}\left(f_{6}\right)_{p} \geq C \frac{\gamma_{m_{n_{k}}}}{\lambda_{m_{n_{k}}}} \geq C C_{n_{k}} \varepsilon_{m_{n_{k}}},
$$

i.e. $f_{6} \notin E_{p}[\varepsilon]$. This contradicts $W_{p}^{\lambda, \beta} \subset E_{p}[\varepsilon]$. The proof of the necessity part in (13) is complete.

STEP 7. We shall prove that $W_{p}^{\lambda, \beta} E[\omega] \subset E_{p}[\varepsilon]$ implies $\frac{\omega_{n}}{\lambda_{n}}=O\left[\varepsilon_{n}\right]$. If the last condition does not hold, then there exists $\left\{C_{n} \uparrow \infty\right\}$ such that $\frac{\omega_{m_{n}}}{\lambda_{m_{n}}} \geq C_{n} \varepsilon_{m_{n}}$. We choose a subsequence $\left\{m_{n_{k}}\right\}$ such that $\frac{m_{n_{k+1}}}{m_{n_{k}}} \geq 2$ and $\omega_{m_{n_{k}}} \geq \frac{1}{2} \omega_{m_{n_{k}}}+\omega_{m_{n_{k+1}}}$. The series

$$
\sum_{k=0}^{\infty} \frac{\omega_{m_{n_{k}}}-\omega_{m_{n_{k+1}}}}{\lambda_{m_{n_{k}}}} \cos \left(m_{n_{k}}+1\right) x
$$

is the Fourier series of a function $f_{16} \in L_{p}$, and $f_{16}^{(\lambda, \beta)} \in W_{p}^{\lambda, \beta} E[\omega]$. On the other hand,

$$
E_{m_{n_{k}}}\left(f_{16}\right)_{p} \geq C \frac{\omega_{m_{n_{k}}}-\omega_{m_{n_{k+1}}}}{\lambda_{m_{n_{k}}}} \geq C \frac{\omega_{m_{n_{k}}}}{\lambda_{m_{n_{k}}}} \geq C C_{n_{k}} \varepsilon_{m_{n_{k}}},
$$

i.e. $f_{16} \notin E_{p}[\varepsilon]$. This contradicts our conjecture $W_{p}^{\lambda, \beta} E[\omega] \subset E_{p}[\varepsilon]$. The proof of the necessity part in (14) is complete.

\section{References}

[Ba1] N. K. Bary, On best approximation of two conjugate functions by trigonometric polynomials, Izv. Akad. Nauk SSSR Ser. Mat. 19 (1955), 285-302.

[Ba2] N. K. Bary, A Treatise on Trigonometric Series, Vol. I, II, Pergamon Press, 1964.

[Be1] S. Bernstein, On the best approximation of continuous functions by polynomials of a given degree, Comm. Soc. Math. Kharkow 13 (1912), 49-194; reprinted in Collected Works (see [Be3]), Vol. I, 11-104; for French transl. see [Be2].

[Be2] S. Bernstein, Sur l'ordre de la meilleure approximation des fonctions continues par les polynomes de degré donné, Mém. Cl. Sci. Acad. Roy. Belg. 4 (1912), 1-103. Available online at http://www.math.technion.ac.il/hat/fpapers/ber2.pdf.

[Be3] S. Bernstein, Collected Works, Vol. I and II, Izdat. Akad. Nauk, Moscow, 1952, 1954.

[Ch-Zh] K. Chen and S. Zhou, On a note of Hasson and Shisha, Soochow J. Math. 17 (1991), 311-315.

[De-Lo] R. A. DeVore and G. G. Lorentz, Constructive Approximation, Springer-Verlag, Berlin, 1993.

[Ge] V. È. Gel̆t, The accuracy of certain inequalities in approximation theory, Math. Notes 10 (1971-72), 768-776; translation from Mat. Zametki 10 (1971), 571-582.

[Ha-Li] G. H. Hardy and J. S. Littlewood, Some properties of fractional integrals, Math. Z. 27 (1928), 565-606. 
[Ha-Sh] M. Hasson and O. Shisha, On the condition $\sum_{n=1}^{\infty} n^{p-1} E_{n}^{*}(f)<\infty$, J. Approx. Theory 39 (1983), 389-393.

$[\mathrm{Ku}] \quad$ D. L. Kudryavtsev, Fourier series of functions that have a fractional-logarithmic derivative, Sov. Math. Dokl. 26 (1982), 311-313; translation from Dokl. Akad. Nauk SSSR 266 (1982), 274-276.

[Kr] D. Králik, Untersuchung der Integrale und Derivierten gebrochener Ordnung mit den Methoden der konstruktiven Funktionentheorie, Acta Math. Acad. Sci. Hungar. 7 (1956), 49-64.

[Mo] F. Moricz, A quantative version of the Young test for the convergence of conjugate series, Journal of Appr. Theory 81 (1995), 207-216.

$[\mathrm{Mu}] \quad$ H. Musielak, On fractional derivatives, Colloq. Math. 27 (1973), 133-139.

[Og] I. I. Ogieveckil, Integration and differentiation of fractional order of periodic functions and the constructive theory of functions, in: Studies of Modern Problems of Constructive Theory of Functions, Fizmatgiz, Moscow, 1961, 159-164.

[Si-Ti] B. Simonov and S. Tikhonov, Embedding theorems for functional classes, available at http://www.arxiv.org/abs/math.CA/0506381.

[Stec] S. B. Stečkin, On best approximation of conjugate functions by trigonometric polynomials, Izv. Akad. Nauk SSSR 20 (1956), 197-206.

[Step] A. I. Stepanets, Inverse theorems for the approximation of periodic functions, Ukraïn. Mat. Zh. 47 (1995), 1266-1273; translation in Ukrainian Math. J. 47 (1996), 14411448 .

[Te] S. A. Telyakovskiı̌, Lower bounds of the integral modulus of continuity of a function in terms of its Fourier coefficients, Math. Notes 52 (1992), 1149-1153; translated from Mat. Zametki 52 (1992), 107-112.

[Ti] A. F. Timan, Theory of Approximation of Functions of a Real Variable, Dover Publications, New York, 1994.

[Zy] A. Zygmund, Trigonometric Series, Vol. I, II, third edition, Cambridge, 2002. 